\title{
Non-thermal recombination - a neglected source of flare hard X-rays and fast electron diagnostic
}

\author{
J. C. Brown and P. C. V. Mallik
}

Department of Physics and Astronomy, University of Glasgow, Glasgow G12 8QQ, UK

e-mail: john@astro.gla.ac.uk

Received 17 June 2007 / Accepted 27 January 2008

\section{ABSTRACT}

\begin{abstract}
Context. Flare Hard X-rays (HXRs) from non-thermal electrons are commonly treated as solely bremsstrahlung (free-free $=\mathrm{f}-\mathrm{f}$ ), recombination (free-bound $=\mathrm{f}-\mathrm{b}$ ) being neglected. This assumption is shown to be substantially in error, especially in hot sources, mainly due to recombination onto Fe ions.

Aims. We analyse the effects on HXR spectra $J(\epsilon)$ and electron diagnostics by including non-thermal recombination onto heavy elements in our model.

Methods. Using Kramers hydrogenic cross sections with effective $Z=Z_{\mathrm{eff}}$, we calculate f-f and f-b spectra for power-law electron spectra within both thin and thick target limits and for Maxwellians with summation over all important ions.

Results. We find that non-thermal electron recombination, especially onto Fe, must, in general, be included with f-f for reliable spectral interpretation, when the HXR source is hot, such as occulted loops containing high ions of Fe (f-b cross-section $\propto Z^{4}$ ). The $\mathrm{f}-\mathrm{b}$ contribution is greatest when the electron spectral index $\delta$ is large and any low energy cut-off $E_{\mathrm{c}}$ is small, because the electron flux spectrum $F(E)$ emitting f-b photon energy $\epsilon$ is $\propto\left(E=\epsilon-V_{Z}\right)^{-\delta}\left(V_{Z}\right.$ is the ionisation potential) and not $\propto(E=\epsilon)^{-\delta+1}$ as for f-f. The f-b spectra recombination edges mean a cut-off $E_{\mathrm{c}}$ in $F(E)$ appears as an HXR feature at $\epsilon=E_{\mathrm{c}}+V_{Z}$, offering an $E_{\mathrm{c}}$ diagnostic. For thick target sources, the presence of $E_{\mathrm{c}}$ appears as edges in $J^{\prime}(\epsilon)$, not in $J(\epsilon)$, but it is still detectable. Including f-b lowers the $F(E)$ needed for prescribed HXR fluxes greatly in some cases; and even when small, it seriously distorts $F(E)$ as inferred by inversion or forward fitting of $J(\epsilon)$ based on f-f alone.

Conclusions. The f-b recombination from non-thermal electrons can be an important contributor to HXR spectra, so it should be included in spectral analyses, especially for hot sources. Accurate results will require use of better cross sections than ours and consideration of source ionisation structure.
\end{abstract}

Key words. atomic processes - Sun: corona - Sun: flares - Sun: X-rays, gamma rays

\section{Introduction}

Ever since their first detection (Arnoldy et al. 1968; Kane \& Andersen 1970), flare hard X-ray (HXR) bursts (photon energies $\epsilon>10 \mathrm{keV}$ or so) have been recognised as an important diagnostic of electron acceleration and propagation (e.g. Brown 1971; Lin \& Schwartz 1987; Johns \& Lin 1992). The large electron flux and power imply they play a substantial role in flare energy budgets and pose challenges for electron acceleration mechanisms (see recent reviews by, e.g. Vilmer et al. 2003; Brown 2005; MacKinnon 2006). Recent copious high resolution HXR spectral data from the RHESSI mission (Lin et al. 2002) have created the possibility of detailed reconstruction of source electron spectra (following Brown 1971) offering important constraints on the electron energy budget and acceleration processes (Piana et al. 2003; Conway et al. 2003; Massone et al. 2004; Kontar et al. 2005, 2006; Brown et al. 2006).

In inferring electron flux spectra $F(E)$, the HXR radiation mechanism has always been taken to be f-f collisional bremsstrahlung of fast electron impacts with atoms and ions, gyrosynchrotron and inverse Compton radiation being negligible at these energies for solar magnetic and radiation fields (Korchak 1971). Though included for thermal electrons in hot (a few keV) plasma, f-b recombination radiation from non-thermals seems to have been assumed negligible other than in a preliminary study by Landini et al. (1973). In view of the importance of details in the photon spectrum $J(\epsilon)$ (photons $\mathrm{s}^{-1} \mathrm{kev}^{-1}$ ) for accurate reconstruction of $F(E)$, we re-examine this assumption, and conclude (cf. Mallik \& Brown 2007) that it is not valid under some conditions, which quite commonly exist in some flare HXR source regions.

It is not the intention of this paper to analyse precisely the theoretical recombination radiation spectrum from fast electrons under conditions (e.g. ionisation structure) for specific flares which are typically both inhomogeneous and time dependent. Rather we give approximate theoretical estimates of how important it may be relative to bremsstrahlung under various limiting conditions. Specifically, we compare the two in the simplest, Kramers, cross-section approximations, for limiting cases of plasma ionisation. The recombination emission rate per electron is very sensitive to the ionic charge, being $\propto Z^{4} A_{Z}$ (Kramers $1923)$ per plasma proton for hydrogenic ions of charge $Z e$ and number abundance $A_{Z}$. Thus the emitted f-b flux and spectrum depend strongly on the ionisation state, hence the temperature, of the plasma where the fast electrons recombine. In practice this will involve several ionisation stages of several target plasma species (since $Z^{4} A_{Z}$ may be large even for small abundance $A_{Z}$ ), which will vary along the paths of the electrons and be time dependent.

The paper is organised as follows. In Sect. 2 we briefly discuss relevant processes and the cross-section approximations we use, and obtain expressions for the total continuum photon 
spectral contributions $j(\epsilon)$ expected from an electron flux spectrum $F(E)$ from f-f and from f-b processes. In Sect. 3, we compare these for a power-law $F(E)$ with low cut off at $E<E_{\mathrm{c}}$ and for a shifted power-law, and discuss implications for flare electron spectra and energy budgets under several limiting plasma ionisation assumptions. In Sect. 4 we look at thermal and nonthermal components to show how the relative importance of each contribution depends on conditions in the flare by varying parameters around those for a specific real event. Section 5 discusses the effect of including the f-b contribution on inverse problem inference of $F(E)$ from $j(\epsilon)$ while Sect. 6 summarises our conclusions and suggests directions for future work. Details of some of the equations are given in Appendix A. In Appendix B we discuss the total emission spectra from extended volumes for thin target, collisional thick target and thermal cases.

\section{Free-free and free-bound emissivity spectra}

\subsection{General considerations}

In this section, we discuss only local emissivities $j(\epsilon)$ (photons $\mathrm{cm}^{-3} \mathrm{~s}^{-1}$ per unit $\left.\epsilon\right)$. Relativistic and directivity effects are disregarded $\left(E, \epsilon \ll m_{\mathrm{e}} c^{2}\right)$ since the $\mathrm{f}$-b/f-f ratio is largest at low $E$. Then, if target atom/ion type $t$ has density $n_{t}$ and the fast electron flux spectrum is $F(E)$ (electrons $\mathrm{s}^{-1} \mathrm{~cm}^{-2}$ per unit $E$ ), $j(\epsilon)$ for a collisional radiation process is

$j(\epsilon)=\Sigma_{t} j_{t}(\epsilon)=\Sigma_{t} n_{t} \int_{E_{\operatorname{tmin}(\epsilon)}}^{\infty} F(E) \frac{\mathrm{d} Q_{t}}{\mathrm{~d} \epsilon}(\epsilon, E) \mathrm{d} E$,

where $\mathrm{d} Q_{t} / \mathrm{d} \epsilon(\epsilon, E)$ is the relevant cross-section per unit $\epsilon$ for target species $t$ and the integral is over the range of electron energies relevant to species $t$.

\subsection{Bremsstrahlung}

In the case of f-f (bremsstrahlung), $\mathrm{d} Q_{t} / \mathrm{d} \epsilon(\epsilon, E)$ is essentially the same for any state of ionisation of an atomic species $Z$ (Koch $\&$ Motz 1959), and the $t$ summation in (1) need only be carried out over elements $Z$ to give, for element abundances $A_{Z}$ (by number relative to hydrogen), and total proton $(\mathrm{p}+\mathrm{H})$ density $n_{\mathrm{p}}$,

$j_{\mathrm{B}}(\epsilon)=n_{\mathrm{p}} \Sigma_{Z} A_{Z} \int_{\epsilon}^{\infty} F(E) \frac{\mathrm{d} Q_{\mathrm{B} Z}}{\mathrm{~d} \epsilon}(\epsilon, E) \mathrm{d} E$,

where $\mathrm{d} Q_{\mathrm{B} Z} / \mathrm{d} \epsilon(\epsilon, E)$ is the bremsstrahlung cross-section for element $Z$ and $E_{\min }=\epsilon$ since any free-free transition can only yield a maximum $\epsilon=E$. The bremsstrahlung cross-section per nucleus $Z$ scales as $Z^{2}$ and can be written

$\frac{\mathrm{d} Q_{\mathrm{B} Z}}{\mathrm{~d} \epsilon}=\frac{8 \alpha r_{\mathrm{e}}^{2} Z^{2}}{3} \frac{m_{\mathrm{e}} c^{2}}{\epsilon E} q(\epsilon, E), \epsilon \leq E$

(and zero for $\epsilon>E$ ). Here $\alpha=e^{2} / \hbar c$ is the fine structure constant and $r_{\mathrm{e}}=e^{2} / m_{\mathrm{e}} c^{2}$ the classical electron radius, while $q(\epsilon, E)$ is the ratio of the actual cross section to the Kramers cross section (Kramers 1923), which is the factor in front of $q$. While this is only a first approximation, not suitable for accurate absolute spectral inversion/reconstruction algorithms (Brown 2005), it will be adequate for the present purpose of comparing $\mathrm{f}-\mathrm{f}$ with f-b emission, which we also treat in the Kramer's approximation. Then (2) and (3) give, for bremsstrahlung,

$j_{\mathrm{B}}(\epsilon)=\frac{8 \alpha r_{\mathrm{e}}^{2}}{3} \frac{m_{\mathrm{e}} c^{2}}{\epsilon} \zeta_{\mathrm{B}} n_{\mathrm{p}} \int_{\epsilon}^{\infty} \frac{F(E)}{E} \mathrm{~d} E$, where

$\zeta_{\mathrm{B}}=\Sigma_{Z} \zeta_{\mathrm{BZ}}=\Sigma_{Z} A_{Z} Z^{2}$

is the heavy element correction for bremsstrahlung, with $\zeta_{\mathrm{B}} \approx$ 1.6 for the solar coronal abundances we use - see later.

\subsection{Recombination radiation}

The situation here is more complicated. Firstly, 2-body radiative recombination (we neglect 3-body recombination) of a free electron of energy $E$ to a bound level $m$ of energy $-V(Z, i, m)$ in ionic stage $i$ yields a photon energy $\epsilon$, which, apart from quantum uncertainty, is unique, namely:

$\epsilon=E+V(Z, i, m)$.

That is, when a fast electron does recombine, all of its kinetic energy $E$ plus $V$ goes into a photon of that energy, in contrast to bremsstrahlung where photons of all energies $\epsilon \leq E$ are emitted.

Furthermore, for each element $Z$, there is a range of $Z+1$ distinct ion stages $i$ each with its own distinct set of energy levels $(m)$ and a set of $Z, i, m$-dependent recombination crosssections. Thus recombination collisions of a mono-energetic beam with a multi-species plasma gives rise to a set of deltafunction-like spectral features at all energies (6) corresponding to elements $Z$, ionic stages $i$ and levels $m$. For a continuous electron spectrum, this yields a continuum photon spectrum that is a sum of an infinite series of energy-shifted electron flux contributions. In contrast to bremsstrahlung it does not involve an integral over a continuum of electron energies.

For a general plasma the basic particle type " $t$ " onto which recombination occurs is level $m$ of ion stage $i$ of element $Z$ with recombination cross-section differential in $\epsilon$ for that $t$ :

$\frac{\mathrm{d} Q_{\mathrm{R} t}}{\mathrm{~d} \epsilon}(\epsilon)=Q_{\mathrm{R} t} \delta\left(E-\epsilon+V_{t}\right)$,

where $Q_{\mathrm{R} t}$ is the total radiative recombination cross-section for species $t$ and $\delta\left(E^{\prime}\right)$ is the delta-function in energy such that $\int_{-\infty}^{\infty} \delta\left(E^{\prime}\right) \mathrm{d} E^{\prime}=1$. Then the total recombination emission spectrum for electron flux spectrum $F(E)$ is

$$
\begin{aligned}
j_{\mathrm{R}}(\epsilon) & =n_{\mathrm{p}} \Sigma_{t} A_{t} \int_{E_{\min }(\epsilon, t)}^{\infty} Q_{\mathrm{R} t}(\epsilon, E) \delta\left(E-\epsilon+V_{t}\right) F(E) \mathrm{d} E \\
& =\Sigma_{t} A_{t} n_{\mathrm{p}} Q_{\mathrm{R} t}\left(\epsilon, \epsilon-V_{t}\right) F\left(\epsilon-V_{t}\right),
\end{aligned}
$$

where $A_{t}$ is the numerical abundance of species $t$ relative to $n_{\mathrm{p}}$. The forms for $Q_{\mathrm{R} t}$, for general $t$, are complicated and have to be calculated numerically, as do the values of $A_{t}$ when individual ionisation states are considered. However, in the Kramers approximation (with unit Gaunt factors) there is an analytic expression for hydrogenic ions, which we will use to estimate $\mathrm{d} j_{\mathrm{R}} / \mathrm{d} \epsilon$ compared with $\mathrm{d} j_{\mathrm{B}} / \mathrm{d} \epsilon$, namely, for recombination onto level $m$ of the hydrogenic ion of element $Z$ (Kramers 1923; Andersen et al. 1992; Hahn 1997)

$Q_{\mathrm{R}}=\frac{32 \pi}{3 \sqrt{3} \alpha} r_{\mathrm{e}}^{2} \frac{Z^{4} \chi^{2}}{m^{3} \epsilon E}$,

where $\chi=m_{\mathrm{e}} e^{4} / 2 \hbar^{2}$ is the hydrogen ionisation potential.

For an element in its highest purely hydrogenic ion state the emissivity spectrum would then be

$j_{\mathrm{R} Z}(\epsilon)=\frac{32 \pi}{3 \sqrt{3} \alpha} \frac{r_{\mathrm{e}}^{2} \chi^{2} Z^{4} n_{z}}{\epsilon} \Sigma_{m} \frac{1}{m^{3}} \frac{F\left(\epsilon-Z^{2} \chi / m^{2}\right)}{\epsilon-Z^{2} \chi / m^{2}}$ 
Table 1. Elements with their coronal abundances and ionisation potentials at $T \gg 10^{8} \mathrm{~K}$.

\begin{tabular}{cccccc}
\hline \hline Element & $Z$ & $A_{z}$ & $A_{z} Z^{2}$ & $A_{z} Z^{4}$ & $V_{z}=Z^{2} \chi(\mathrm{keV})$ \\
\hline $\mathrm{H}$ & 1 & 1 & 1 & 1 & 0.0136 \\
$\mathrm{He}$ & 2 & 0.096 & 0.384 & 1.536 & 0.0544 \\
$\mathrm{C}$ & 6 & $3.57 \times 10^{-4}$ & 0.013 & 0.463 & 0.490 \\
$\mathrm{O}$ & 8 & $8.57 \times 10^{-4}$ & 0.055 & 3.511 & 0.870 \\
$\mathrm{Ne}$ & 10 & $1.07 \times 10^{-4}$ & 0.011 & 1.071 & 1.360 \\
$\mathrm{Mg}$ & 12 & $1.33 \times 10^{-4}$ & 0.019 & 2.755 & 1.958 \\
$\mathrm{Si}$ & 14 & $1.27 \times 10^{-4}$ & 0.025 & 4.871 & 2.666 \\
$\mathrm{~S}$ & 16 & $1.61 \times 10^{-5}$ & 0.0041 & 1.053 & 3.482 \\
$\mathrm{Ca}$ & 20 & $8.50 \times 10^{-6}$ & 0.0034 & 1.360 & 5.440 \\
$\mathrm{Fe}$ & 26 & $8.61 \times 10^{-5}$ & 0.058 & 39.336 & 9.914 \\
$\mathrm{Ni}$ & 28 & $6.95 \times 10^{-6}$ & 0.0054 & 4.27 & 10.662 \\
\hline \multicolumn{5}{c}{$\Sigma=1.58$} & $\Sigma=61.2$ \\
\hline
\end{tabular}

with the $m$ summation over $m \geq Z(\chi / \epsilon)^{1 / 2}$, since recombination to level $m$ yields only photons of $\epsilon \geq Z^{2} \chi / \mathrm{m}^{2}$. If the source were so hot that all atoms were almost fully ionised the total for all $Z$ would be, in this approximation,

$j_{\mathrm{R}}(\epsilon)=\frac{32 \pi}{3 \sqrt{3} \alpha} \frac{r_{\mathrm{e}}^{2} \chi^{2}}{\epsilon} n_{\mathrm{P}} \Sigma_{Z} Z^{4} A_{Z} \Sigma_{m} \frac{1}{m^{3}} \frac{F\left(\epsilon-Z^{2} \chi / m^{2}\right)}{\epsilon-Z^{2} \chi / m^{2}}$

for element abundances $A_{Z}$, with the same $m$ summation limits.

In reality even super-hot coronal flare temperatures are not high enough to equal the ultra-hot $T \gg 10^{8} \mathrm{~K}$ needed to almost fully ionise all elements into their hydrogenic states, especially $\mathrm{Fe}$, which is crucial in having by far the highest value of $A_{Z} Z^{4}$ - see Table 1. Consequently, to deal accurately with $j_{R}$ for real flare data, we would have to take into account the actual ionisation state of the flare plasma, which varies with time and location (being radically different in loop tops from loop footpoints), and actual forms of $Q_{\mathrm{R}}(Z), V_{Z}$ for non-hydrogenic ion stages.

For our purpose of making first estimates we make the following simplifying approximations:

- We treat all ions using hydrogenic Eqs. (9)-(11) but with suitably chosen $Z_{\text {eff }}$ so that

$$
V_{Z}=Z_{\mathrm{eff}}^{2} \chi ; \quad Q_{\mathrm{R} Z}=\frac{32 \pi}{3 \sqrt{3} \alpha} r_{\mathrm{e}}^{2} \frac{Z_{\mathrm{eff}}^{4} \chi^{2}}{m^{3} \epsilon E},
$$

where $Z_{\text {eff }}$ makes allowance for screening and other nonhydrogenic effects. While this will be a rough estimate for some ions, such approximations are often quite satisfactory for suitable $Z_{\text {eff }}$ (e.g. Hahn \& Krstic 1994; Erdas et al. 1993). Here we adopt $Z_{\text {eff }}$ such that hydrogenic Eq. (12) gives the correct value of $Q_{\mathrm{R} Z}$ as given by exact calculations such as those of Arnaud \& Raymond (1992) for Fe, which is the most important ion in our analysis. Typically, for an element of atomic number $Z$ in an ionic state with $z$ bound electrons left, $Z_{\text {eff }}$ is between $Z-z$ and $Z-z+1$.

- Noting that $Q_{\mathrm{R}} \propto 1 / \mathrm{m}^{3}$ we include here only recombination to $m=1$ (in the sense of the lowest empty level of the ion - hydrogenic with $Z=Z_{\text {eff }}-$ not of the atom). Higher $m$ contributions are weaker, being $\propto 1 / \mathrm{m}^{3}$ though extending to lower energies with edges at $Z_{\text {eff }}^{2} \chi / m^{2}$. These should be included in quantitative data fitting.

- We focus on situations where the emitting region is near isothermal and either quite cool, so that only low $V_{Z}$ element recombination matters, or very hot so that high $V_{Z}$ elements (mainly $\mathrm{Fe}$ ) are dominant. The former are typically
Table 2. Ionic species of iron at 20 MK.

\begin{tabular}{ccccccc}
\hline \hline Element & $Z-z$ & $Z_{\text {eff }}$ & $\Phi_{Z_{\text {eff }}}$ & $A_{z}$ & $A_{z} Z_{\text {eff }}^{4}$ & $V_{z}=Z_{\text {eff }}^{2} \chi(\mathrm{keV})$ \\
\hline Fe XXII & 21 & 21.98 & 0.05 & $0.43 \times 10^{-5}$ & 1.004 & 6.57 \\
Fe XXIII & 22 & 22.61 & 0.14 & $1.21 \times 10^{-5}$ & 3.152 & 6.95 \\
Fe XXIV & 23 & 23.20 & 0.25 & $2.15 \times 10^{-5}$ & 6.232 & 7.32 \\
Fe XXV & 24 & 23.77 & 0.56 & $4.82 \times 10^{-5}$ & 15.381 & 7.68 \\
\hline
\end{tabular}

loop chromospheric footpoints (thick target) and the latter very hot coronal loops which are either at the limb with their footpoints occulted, or are so dense as to be coronal thick targets (Veronig \& Brown 2004).

Under these conditions, Eq. (11) becomes

$j_{\mathrm{R}}(\epsilon)=\frac{32 \pi}{3 \sqrt{3} \alpha} \frac{r_{\mathrm{e}}^{2} \chi^{2}}{\epsilon} n_{\mathrm{p}} \Sigma_{Z_{\mathrm{eff}}} Z_{\mathrm{eff}}^{4} A_{Z_{\mathrm{eff}}} \frac{F\left(\epsilon-Z_{\mathrm{eff}}^{2} \chi\right)}{\epsilon-Z_{\mathrm{eff}}^{2} \chi}$,

where $A_{Z_{\text {eff }}}=A_{Z} \Phi_{Z_{\text {eff }}}$ with $\Phi_{Z_{\text {eff }}}$ the fraction of atoms of element $Z$ in ionic state $Z_{\text {eff }}$.

Note that, since there is no integration over $E$ here, if $F(E)$ contains a sharp feature at an electron energy $E_{*}$, such as a low or high $E$ cut-off, this will appear in the recombination contribution to the photon spectrum $j(\epsilon)$ as a series of sharp features at photon energies $\epsilon\left(m, Z, E_{*}\right)=E_{*}+Z_{\text {eff }}^{2} \chi / m^{2} ; m=1, \infty$ for every ion $Z$ present. The same is true for broad features like smooth bumps or dips. This is in contrast with the bremsstrahlung contribution, in which such features are smoothed out by integration over $E$. Thus, even if $j_{\mathrm{R}} \ll j_{\mathrm{B}}$, it may have an important effect in inferring $F(E)$ from $j(\epsilon)$ since this essentially involves differentiating $j(\epsilon)$ (Sect. 5).

\subsection{Element parameters and flare plasma ionisation}

The heavy element correction for bremsstrahlung, $\zeta_{\mathrm{B}}$, is almost independent of ionisation state (since the bremsstrahlung cross sections for atoms and ions of the same $Z$ are essentially the same), being $\zeta_{\mathrm{B}} \approx 1.6$ for solar abundances. On the other hand $\zeta_{\mathrm{R} Z_{\mathrm{eff}}}=Z_{\mathrm{eff}}^{4} A_{Z_{\mathrm{eff}}}$ depends on the number of empty ion levels available for recombination. The importance of fast electron recombination radiation thus depends on the state of ionisation of the plasma in which the fast electrons are moving, which is primarily a function of plasma temperature $T$.

In Table 1 we show the values of $Z, Z^{2} A_{Z}=\zeta_{\mathrm{B} Z}, Z^{4} A_{Z}=\zeta_{\mathrm{R} Z}$, $V_{Z}$ for various elements/ions whose $\zeta_{\mathrm{R} Z}=Z^{4} A_{Z}$ is large enough to be significant, if the element is sufficiently ionised. With $\zeta_{\mathrm{RZ}} \approx 40$ for FeXXVI, Fe is by far the most important if conditions are such that it is highly ionised. The $k T$ where maximum ionisation of an ion stage is reached is typically of the order $0.1 Z_{\text {eff }}^{2} \chi$ to $Z_{\text {eff }}^{2} \chi$. In Table 2 we show more detailed values for several stages of ionisation of Fe (XXII-XXV, i.e. $21+$ to $24+$ ) with the appropriate $A_{Z_{\mathrm{eff}}}=A_{Z_{\mathrm{eff}}} \Phi_{Z_{\mathrm{eff}}}$ for each of these Fe ionic states for the typical coronal flare case of $T=2 \times 10^{7} \mathrm{~K}$. These are taken from Arnaud \& Raymond (1992) as are the actual ionisation fractions we adopt later (Sect. 4) for the temperatures of the real flare we consider.

The radiative recombination coefficients give $Z_{\mathrm{eff}}$, which differ slightly from the $Z$ values, as mentioned in Sect. 2.3. For the 2002 April 14 event, to which we return later, the peak flare temperature was $19.6 \mathrm{MK}, \sim 5 \%$ of the iron appearing as Fe XXII $\left(\mathrm{Fe}^{21+}\right), \sim 14 \%$ in the Fe XXIII $\left(\mathrm{Fe}^{22+}\right)$ state, $\sim 25 \%$ appearing as Fe XXIV and $\sim 56 \%$ as Fe XXV. The respective $Z_{\text {eff }}$ values are $21.98,22.61,23.20$ and 23.77. 
Broadly speaking in typical flare/micro-flare conditions we can consider the following $T$ regimes:

- at $T \leq 10^{4} \mathrm{~K}$ ("cold") even $\mathrm{H}$ and other low $V_{Z_{\text {eff }}}$ ions are neutral so $\zeta_{\mathrm{RZ}} \approx 0$ for all $Z$. This would be typical of very dense cool chromospheric thick target footpoints relevant to deeply penetrating electrons;

- for $10^{5} \leq T \leq 10^{6} \mathrm{~K}$ ("cool") the predominant elements ionised are $\mathrm{H}, \mathrm{O}, \mathrm{Mg}, \mathrm{Si}$ giving $\Sigma_{Z} \zeta_{\mathrm{RZ}} \approx 15$. This is most relevant to upper chromospheric dense warm plasma reached by moderate energy thick target electrons;

- at $T \geq 10^{7} \mathrm{~K}$ ("hot") Fe is well ionised up to about Fe XXV giving $\Sigma_{Z} \zeta_{\mathrm{R} Z} \approx 50$.

This is relevant to the hot "coronal" loop regime, hence either to (i) typical upper (SXR) flare loops of moderate density (thin target) whose HXR emission is seen in isolation either by HXR spectroscopic imaging or volume integrated but with the cool footpoints occulted because they are over the solar limb; or (ii) cases of coronal thick target loops (Veronig \& Brown 2004) where the upper loop density suffices to stop the fast electrons collisionally.

\section{Local (thin target) HXR spectra of $f-f$ and $f-b$ for power-law $F(E)$ with cut-off}

\subsection{Basic expressions for $j_{B}, j_{R}$}

To estimate how the fast electron recombination $j_{\mathrm{R}}(\epsilon)$ compares with bremsstrahlung $j_{\mathrm{R}}(\epsilon)$, we first consider the commonly studied case of a power-law with a low energy cut-off

$F(E)=(\delta-1) \frac{F_{\mathrm{c}}}{E_{\mathrm{c}}}\left(\frac{E}{E_{\mathrm{c}}}\right)^{-\delta} ; E \geq E_{\mathrm{c}}$,

where $F_{\mathrm{c}}$ is the total electron flux at $E \geq E_{\mathrm{c}}$. Then, from Eqs. (4) and (14), we obtain for f-f emission

$$
\begin{aligned}
j_{\mathrm{B}}(\epsilon)= & \frac{\delta-1}{\delta} \frac{8 \alpha \zeta_{\mathrm{B}}}{3} \frac{m_{\mathrm{e}} c^{2} r_{\mathrm{e}}^{2}}{\epsilon} \frac{n_{\mathrm{p}} F_{\mathrm{c}}}{E_{\mathrm{c}}} \\
& \times\left[\frac{\epsilon}{E_{\mathrm{c}}}\right]^{-\delta} ; \epsilon \geq E_{\mathrm{c}} \\
& \times 1 ; \epsilon<E_{\mathrm{c}},
\end{aligned}
$$

while for $\mathrm{f}-\mathrm{b}$ emission from an ion of effective charge $Z_{\mathrm{eff}}$,

$$
\begin{aligned}
j_{\mathrm{R} Z_{\mathrm{eff}}}(\epsilon)= & (\delta-1) \frac{32 \pi \zeta_{\mathrm{R} Z_{\mathrm{eff}}}}{3^{3 / 2} \alpha} \frac{r_{\mathrm{e}}^{2} \chi^{2}}{\epsilon} \frac{n_{\mathrm{p}} F_{\mathrm{c}}}{E_{\mathrm{c}}^{2}} \\
& \times\left[\frac{\epsilon-Z_{\mathrm{eff}}^{2} \chi}{E_{\mathrm{c}}}\right]^{-\delta-1} ; \epsilon \geq E_{\mathrm{c}}+Z_{\mathrm{eff}}^{2} \chi \\
& \times 0 ; \epsilon<E_{\mathrm{c}}+Z_{\mathrm{eff}}^{2} \chi,
\end{aligned}
$$

where

$$
\zeta_{\mathrm{R} Z_{\mathrm{eff}}}=A_{Z_{\mathrm{eff}}} Z_{\mathrm{eff}}^{4} \text {. }
$$

So the total for all relevant $V_{Z_{\text {eff }}}$ is

$j_{\mathrm{R}}(\epsilon)=\Sigma_{Z_{\mathrm{eff}} \geq\left[\left(\epsilon-E_{\mathrm{c}}\right) / \chi\right]^{1 / 2}} j_{\mathrm{R} Z_{\mathrm{eff}}}(\epsilon)$.

\subsection{Ratio of $j_{R}$ to $j_{B}$}

For this truncated power-law case, the ratio of $f-b$ to $f-f$ emissivity is

$$
\begin{aligned}
\Psi & =\frac{j_{\mathrm{R}}(\epsilon)}{j_{\mathrm{B}}(\epsilon)} \frac{2 \pi \delta}{\sqrt{3}} \frac{\chi}{\epsilon} \Sigma_{\left.Z_{\mathrm{eff}}^{2}>\left(\epsilon-E_{\mathrm{c}}\right) / \chi\right)} \frac{\zeta_{\mathrm{R} Z_{\mathrm{eff}}}}{\zeta_{\mathrm{B}}}\left[1-\frac{Z_{\mathrm{eff}}^{2} \chi}{\epsilon}\right]^{-\delta-1} \\
& \approx \frac{0.25(\delta / 5)}{\epsilon(\mathrm{keV})} \Sigma_{\left.Z_{\mathrm{eff}}^{2}>\left(\epsilon-E_{\mathrm{c}}\right) / \chi\right)} \frac{\zeta_{\mathrm{R} Z_{\mathrm{eff}}}}{\zeta_{\mathrm{B}}}\left[1-\frac{Z_{\mathrm{eff}}^{2} \chi}{\epsilon}\right]^{-\delta-1},
\end{aligned}
$$

where each term in the summation is zero at $\epsilon<E_{\mathrm{c}}+Z_{\mathrm{eff}}^{2} \chi$.

For $\epsilon \gg E_{\mathrm{c}}, \Psi \rightarrow 0.25 \Sigma_{Z_{\mathrm{eff}}} A_{Z_{\mathrm{eff}}} Z_{\mathrm{eff}}^{4} / \epsilon(\mathrm{keV})$. In pure ionised $\mathrm{H}$ $\left(\Sigma_{Z} \zeta_{\mathrm{RZ}}=1\right)$ this is only $2.5 \%$ at $10 \mathrm{keV}$. This rather small value of $\Psi$ must be the origin of the conventional wisdom that f-b can be ignored compared to $\mathrm{f}-\mathrm{f}$ emission at HXR energies. However, this notion neglects several crucial facts:

- At high coronal flare temperatures, where all elements are highly ionised, in plasmas of cosmic chemical abundances, heavy elements are the main contributors to the $A_{Z} Z^{4}$ sum. For the extreme ultra-hot case of near-total ionisation of all $Z$, and for modern solar coronal abundances the $\Sigma_{Z}$ factor is $\approx 61.2$, mainly due to Fe as discussed in Sect. 2.4 - see Tables 1 and 2. Note that Fe coronal abundance, for example, has been assumed to be 2.9 times photospheric Fe abundance (Feldman et al. 1992). Even higher factors of about 4 have been suggested (Dennis, personal communication).

- At lower $\epsilon$ the contribution from each $Z_{\text {eff }}$ rises steeply to a sharp recombination edge at $\epsilon=E_{\mathrm{c}}+V_{Z}$, where the flux can be large, especially if $E_{\mathrm{c}}$ is small and $\delta$ large.

- At the edge, the [ ] factor in Eq. (19) goes to $\left[1+Z_{\mathrm{eff}}^{2} \chi / E_{\mathrm{c}}\right]^{\delta+1}$. This is because the flux of electrons emitting recombination photons of energy $\epsilon$ is not the flux of those at $E \geq \epsilon$, as for bremsstrahlung, but of those at $E=\epsilon-Z_{\text {eff }}^{2} \chi$. Consequently $\Psi$ is not negligible even at $\epsilon \gg E_{\mathrm{c}}$. For fully ionised $\mathrm{Fe}$ alone, this factor is $\approx\left[1+10 / E_{\mathrm{c}}(\mathrm{keV})\right]^{\delta+1}$, which, for $\delta=5$ and at $\epsilon=10 \mathrm{keV}$, is $64,11.4,5.5$ for $E_{\mathrm{c}}=10,20,30 \mathrm{keV}$ respectively. Even for lower stage Fe ions (e.g. XXV), common in flare coronal loops, evidently recombination must be a significant contributor to the HXR emission in those parts of the flare.

\subsection{Typical results in limiting regimes}

N.B. All spectrum figures in this paper (except Fig. 5) have been plotted for a bin-width of $1 \mathrm{keV}$ to match RHESSI's spectral resolution. However, in Fig. 5 we use $0.01 \mathrm{keV}$ resolution so as to compare it with Plot A of Fig. 4 to see how f-b edges would look if they were observed at a higher resolution. The $1 \mathrm{keV}$ binning smears out a lot of the edges of different elements that are clearly noticeable in Fig. 5. Hence in Figs. 3 and 4, the edges are not "infinitely" steep as they should be; this is evident in Fig. 5 where they do look "infinitely" steep due to the finer resolution. Also important to note is that the features seen in Figs. 3-5 are recombination edges and not spectral lines. None of the figures in this paper includes spectral lines - leaving them out shows more clearly where f-b edges exist in the HXR continuum.

In Fig. 1 we show for $\delta=3,5$ the actual spectral shapes for $E_{\mathrm{c}}=10,25 \mathrm{keV}$ respectively in plasmas of normal solar coronal abundances, which are: ultra-hot $\left(T \gg 10^{8} \mathrm{~K}\right.$; Fe is nearly fully ionised), hot ( $T=2 \times 10^{7} \mathrm{~K}$; Fe well ionised up to Fe XXV) and $\operatorname{cool}\left(T=10^{6} \mathrm{~K}\right.$; elements up to $\mathrm{Si}$ are almost fully ionised). In Fig. 2 we show the ratios $\Psi(\epsilon)$ for the ultra-hot, hot and "cool" cases, respectively. The following key features of the hot thin target situation are apparent from these figures:

- The peak non-thermal f-b contribution, in each hot or ultrahot case shown, adds at least $50 \%$ to the usual $\mathrm{f}-\mathrm{f}$ one and in some cases $\left(\delta=5, E_{\mathrm{c}}=10 \mathrm{keV}\right)$ is up to 10 times greater ( $1000 \%$ increase) even when only ions up to Fe XXV are present. This is essentially due to the high abundance of $\mathrm{Fe}-$ much higher than thought when recombination spectra were first discussed (Culhane 1969; Culhane \& Acton 1970). In Appendix A we evaluate the efficiency with which 
Non-thermal (NT) f-f, $f-b$ and total spectral shapes
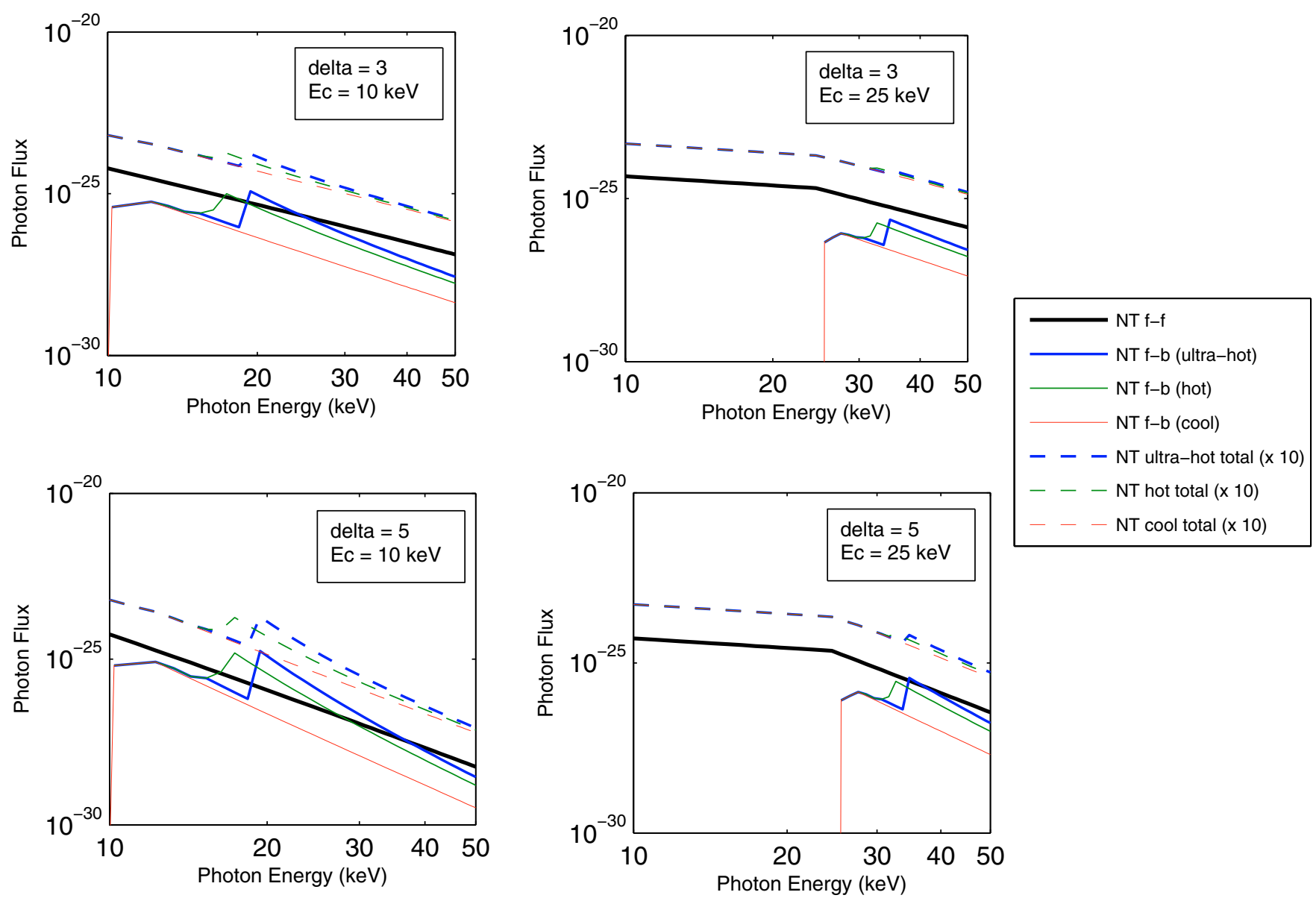

Fig. 1. Actual shapes of non-thermal f-b and f-f spectra for different temperature regimes and non-thermal electron parameters. Note that the cool, hot and ultra-hot totals are almost identical and the dashed curves nearly indistinguishable for $E_{\mathrm{c}}=25 \mathrm{keV}$.

f-b yields HXRs compared to f-f, and also derive the ratio $\Psi$ for the case of a smooth $F(E)$ with no cut-off. This proves, that in a hot enough plasma, far less electrons and power are needed than is found when only $\mathrm{f}-\mathrm{f}$ is included and that, for smooth $F(E), \Psi$ is largest for large $\delta$ and low $E$ spectral rollover.

- In the "cool" case $\left(T \approx 10^{6} \mathrm{~K}\right)$ of elements up to Si almost fully ionised, the f-b contribution is smaller but not in general negligible. For example, in the bottom left panel of Fig. 1 $\left(\delta=5, E_{\mathrm{c}}=10 \mathrm{keV}\right), \mathrm{f}-\mathrm{b}$ is about $30 \%$ of $\mathrm{f}-\mathrm{f}$ at $15 \mathrm{keV}$ energies. This is amply large enough to have a major impact on inferring $F(E)$ by inversion or by forward fitting (Sect. 5).

- In hot plasma, Fe is by far the most important contributor of recombination radiation.

- The peak ratio of $\mathrm{f}-\mathrm{b}$ to $\mathrm{f}-\mathrm{f}$ increases as $\delta$ is increased and/or $E_{\mathrm{c}}$ is decreased. This is because f-b photons of energy $\epsilon$ are emitted by electrons of energy $E-V$ which have flux $F(E-$ $V) \propto(E-V)^{-\delta}$ which is greatest when the minimum $E=E_{\mathrm{c}}$ is smallest, $V$ is largest and the steepness $\delta$ greatest.

- Recombination edges are apparent for the elements with the highest values of $A_{Z_{\text {eff }}} Z_{\text {eff }}^{4}-\mathrm{Fe}, \mathrm{Si}, \mathrm{Mg}$ and $\mathrm{O}$ and at energies $\epsilon=E_{\mathrm{c}}+Z_{\mathrm{eff}}^{2} \chi$, thereby creating the possibility of finding the location of a low energy cut-off $E_{\mathrm{c}}$ should one exist.

- The harder asymptotic $\gamma=\delta+1$ for f-f compared with $\gamma=\delta+2$ for $\mathrm{f}-\mathrm{b}$ (Eqs. (15) and (16)) results in an upward "knee" in the total spectrum clearly visible in Fig. 1 for $E_{\mathrm{c}}=10 \mathrm{keV}$ but also present for higher $E_{\mathrm{c}}$ outside the $\epsilon$ range of the figure. This could be an important signature in data of a substantial f-b contribution.

While the edge locations and the spectral shape trends will be roughly right, our use of the hydrogenic and $Z_{\mathrm{eff}}$ approximations, and adoption of unit Gaunt factors, mean that these curves/analytic forms can only be used for approximate quantitative fitting of real data. As far as we are aware (Kaastra, personal communication) the Gaunt factors, rates etc. have only ever been systematically evaluated for Maxwellian $F(E)$ and sometimes for forms which can be written as sums of these (such as pure power-laws with no cut-off), and some occasional consideration of specific non-thermal spectra (e.g. Landini et al. 1973). Comparison of our Maxwellian results, in the unit Gaunt factor Kramers approximation, with those of Culhane for the same parameters shows the necessary corrections in the Maxwellian case to be significant for quantitative comparison with real data. In addition, in real cases the non-thermal emission will always be superposed on thermal contributions (especially important for the very hot plasmas of special interest here) and also in many cases on a thick target non-thermal contribution (unless this is from occulted footpoints), from the flare volume as a whole. In Appendix B we derive the generalisation of the above equations to the various cases involved in real flares, viz. finite volume thin targets, Maxwellian plasmas and thick targets for use in Sect. 4, where we evaluate the sum of all these contributions for a specific case. 
Non-thermal ratios $f-b: f-f$
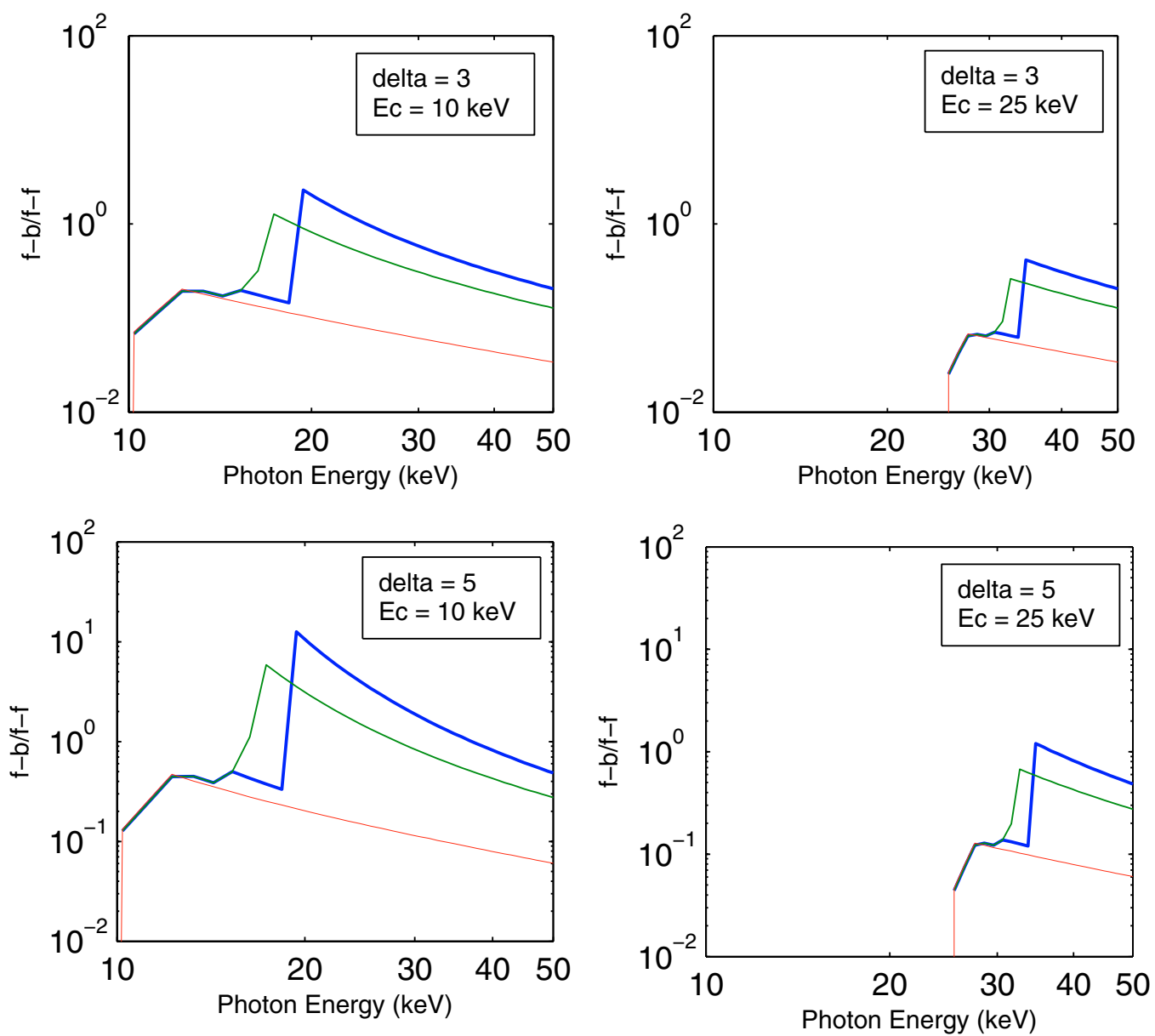

Fig. 2. Photon flux ratio of non-thermal f-b to f-f emission for different temperature regimes and parameters. Line styles have the same meaning as in Fig. 1.
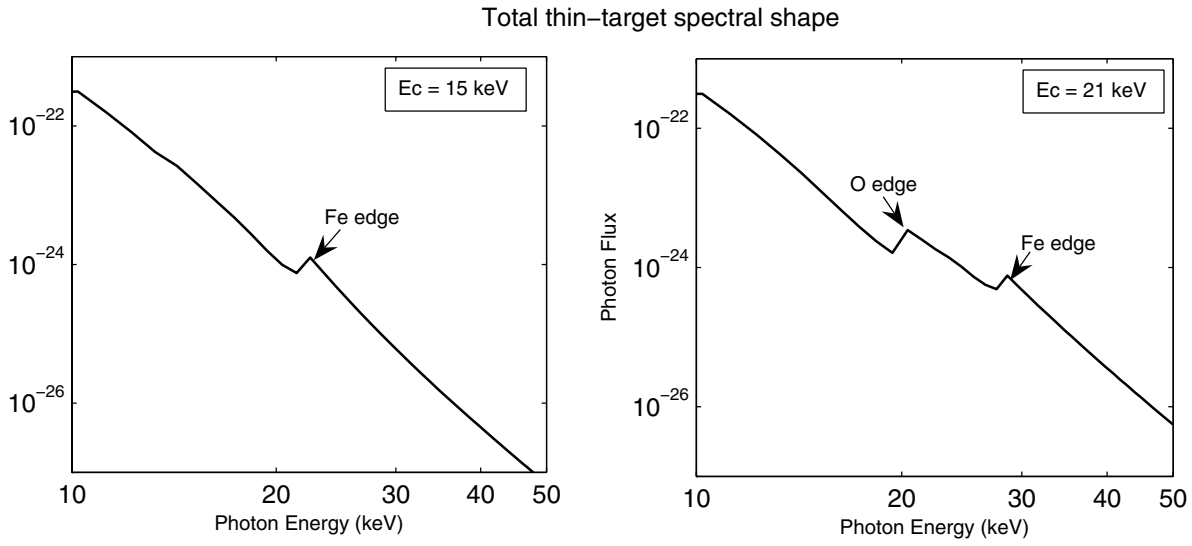

Fig. 3. Spatially localised spectra from a loop with the 2002 April 14 event plasma parameters for two values of $E_{\mathrm{c}}$. The left plot shows a very distinct iron edge at $\approx 22 \mathrm{keV}\left(=E_{\mathrm{c}}+V_{\mathrm{Fe} 24+}\right)$ and a much less predominant oxygen edge at $\approx 15 \mathrm{keV}\left(=E_{\mathrm{c}}\right)$, whereas the second plot shows very distinct oxygen $\left(\approx 21 \mathrm{keV}\left(=E_{\mathrm{c}}\right)\right)$ and iron $(\approx 28 \mathrm{keV})$ edges. This shows the value of recombination as an $E_{\mathrm{c}}$ diagnostic. The "edges" appear to be of finite slope because of the finite $(1 \mathrm{keV})$ resolution used.

\section{Some practical case study results derived from a real flare}

We saw above and in the appendices that the most favourable conditions for a substantial recombination contribution are when the maximum possible amount of the observable HXR source is a hot plasma (e.g. loop) at SXR temperatures. High density maximises the emission measure but may make the source/loop collisionally thick and smear recombination edge spectral signatures of low energy cut offs. So an optimal case could be a loop which is just tenuous enough to be collisionally thin and for which the cool dense thick target footpoints are occulted. (Footpoint removal by imaging is limited by RHESSI's dynamic range). Such sources will have a strong HXR source in the coronal loop. One such event was adopted as a basis for a case study, starting from the real event parameters. This was the 2002 April 14 event, which Veronig \& Brown (2004) showed to be a hot, dense, collisionally thick loop with a strong coronal HXR source and no footpoints up to at least $60 \mathrm{keV}$. Thus the hot coronal source of non-thermal f-b emission was not diluted by cold footpoint thick target $\mathrm{f}-\mathrm{f}$ emission though the $\mathrm{f}-\mathrm{b}$ edges were smeared because 


\section{Spectral shapes of $f-f$ and $f-b$ by varying parameters of a real event}

A

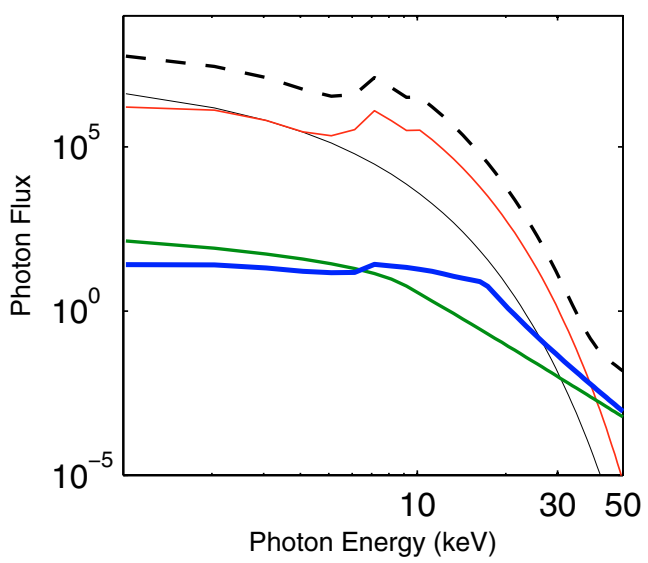

C

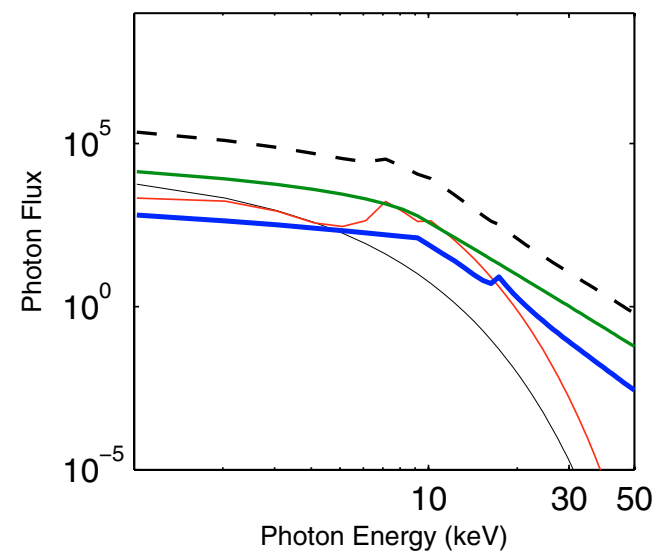

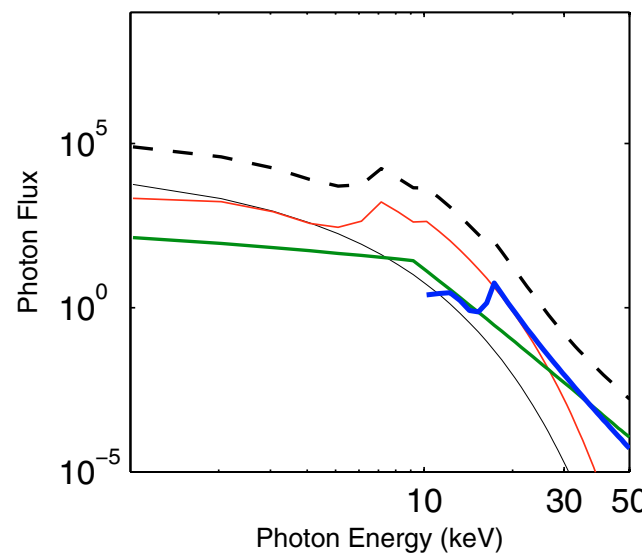

$-f-f$ thermal
$f-b$ thermal
$f-f$ non-thermal
$f-b$ non-thermal
--- total $(x 10)$

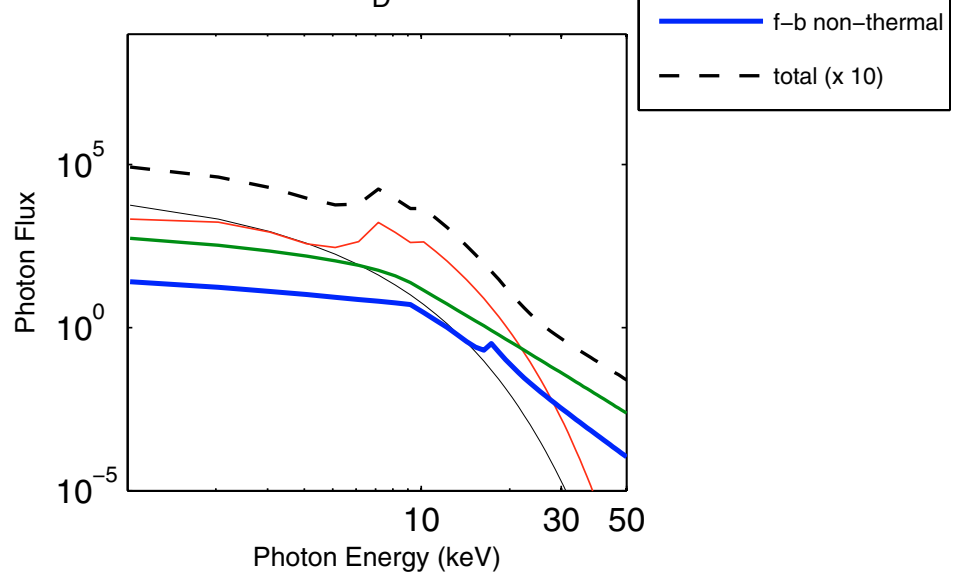

Fig. 4. The spectral components for 4 different hypothetical situations. We show these spectra by varying the parameters around the results in the Veronig \& Brown (2004) paper that analyses the coronal thick target 2002 April 14 event. In all cases we keep the same values of $\delta=6.7$, $E_{\mathrm{c}}=10 \mathrm{keV}$ and $T=19.6 \mathrm{MK}$. Plot A is for the thick-target coronal case with the actual event parameters $n_{\mathrm{p}}, \mathcal{F}_{\mathrm{oc}}$ according to Veronig and Brown. Plot B was obtained for the same event parameters but with $n_{\mathrm{p}}$ reduced 25 times to make the loop collisionally thin above $10 \mathrm{keV}$ and with footpoint emission occulted. The injection rate is the same as Plot A so the density fraction of fast electrons is 25 times higher. The non-thermal emission is down by 25 times while the thermal is down by a factor of 625 . Plot $\mathrm{C}$ is the same as B but with cold thick target footpoints included. The cold footpoint emission (motsly f-f) is dominant. Plot D is the same as C, but with an injection rate reduced by a factor of 25 so that the density fraction of fast electrons is the same as in Plot A. Evidently the detectability of the $\mathrm{f}-\mathrm{b}$ contribution and of associated features in $F(E)$ is sensitive to plasma parameters and observing conditions/geometry.

the hot loop itself slowed the fast electrons to rest. In Fig. 3 we show the theoretical spectrum from a hypothetical resolved part of the coronal loop for two $E_{\mathrm{c}}$ values. We have evaluated the theoretical thermal, non-thermal and the whole volume hypothetical total $J_{\mathrm{B}}(\epsilon), J_{\mathrm{R}}(\epsilon)$ (from Sects. 2, 3 and Appendix B) for such a loop, based on our approximate Kramers expressions, in three loop parameter regimes (Fig. 4):

- Plot A: With the actual hot thick target loop parameters found by Veronig and Brown, namely $\delta=6.7 ; T=19.6 \mathrm{MK}$; $L=45 \times 10^{8} \mathrm{~cm} ; A=19.1 \times 10^{16} \mathrm{~cm}^{2} ; n_{\mathrm{p}}=10^{11} \mathrm{~cm}^{-3}$; $N=4.9 \times 10^{20} \mathrm{~cm}^{-2} ; \mathcal{F}_{1}=5 \times 10^{35} \mathrm{~s}^{-1}$ above $E_{1}=25 \mathrm{keV}$. The total $J$ is dominated by thermal f-b and f-f at low $\epsilon$ but thick-target $\mathrm{f}-\mathrm{b}$ at medium $\epsilon$ and thick-target $\mathrm{f}-\mathrm{f}$ at high $\epsilon$. Locally within the loop volume, if this were spatially resolved, the spectrum $j$ would be like those in Fig. 3, where edges are clearly visible in positions corresponding to cutoff energies of 15 and $21 \mathrm{keV}$. At a higher resolution, these edges would look similar to the edges shown in Fig. 5. Should such edges be found in data, they can diagnose the all-important $E_{\mathrm{c}}$ parameter.
- Plot B: With the actual parameters found by Veronig and Brown except with $n_{\mathrm{p}}$ reduced by a factor of 25 so that the loop is collisionally thin above about $10 \mathrm{keV}$ but with the footpoints hidden (limb occulted) so there is no cold thick target contribution. In this case the thermal emission is also much reduced because $E M=2 n_{\mathrm{p}}^{2} A L$ is down by a factor of 625 . Somewhere between this and the first case should be the optimum condition for seeing maximum $\mathrm{f}-\mathrm{b}$ contribution.

- Plot C: The same as B but with the dominant cold footpoint thick target emission added to show its diluting effect.

- Plot D: The same as $\mathrm{C}$ but with a reduced injection rate and so the thermal is more dominant than in $\mathrm{C}$ and this alters the total spectral shape a little bit.

The upward "knee" apparent in Figs. 4A, B at around $40 \mathrm{keV}$ due to the transition from a f-b to a f-f dominated spectrum (cf. Sect. 3 and Fig. 3) is rarely seen in data but may be present in some events (Conway et al. 2003). A statistical survey of a large sample of events should shed light on conditions where non-thermal f-b is important. Also note that an upward "knee" 


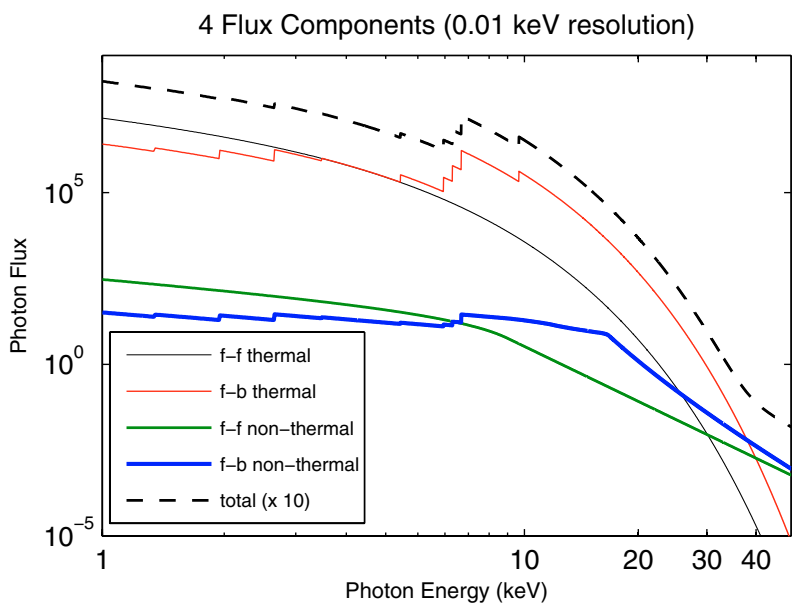

Fig. 5. Spectral components for a resolution of $0.01 \mathrm{keV}$. The f-b edges of all elements involved are clearly noticeable. The parameters are $T=$ 19.6 MK, $E_{\mathrm{c}}=10 \mathrm{keV}$ and $\delta=6.7$. This plot can be compared to Plot A of Fig. 4, which has the same parameters but for $1 \mathrm{keV}$ binning resolution.

is present at the transition from a thermal- to a non-thermaldominated spectrum. The position of this knee depends on the plasma temperature and may interfere with the f-b to f-f "knee", which depends mainly on the $E_{\mathrm{c}}$ parameter. Hence, although for certain parametric conditions one may be able to notice two separate upward "knees", if $E_{\mathrm{c}}$ is low and $T$ is high, the "knees" may occur at similar $\epsilon$ and may not be distinguishable in real data.

\section{The inverse problem - effect of f-f on $F(E)$ inferred from data on $j(\epsilon)$}

We note again that, since even the thin target $j_{\mathrm{B}}$ involves an integral over $E$ while $j_{\mathrm{R}}$ does not, any sharp features in $F(E)$ would be smoothed out in the bremsstrahlung contribution to the photon spectrum but not in the recombination contribution. Consequently, an important way to study the effect of including $\mathrm{f}-\mathrm{b}$ on the required properties of $F(E)$ is to consider it as an inverse problem (Craig \& Brown 1986) to infer $F(E)$ from observed $j(\epsilon)$. Here we consider the following experiment for the thin target case. (Thick target and thermal cases always involve even greater error magnification - Brown \& Emslie 1988). Generate the total $j(\epsilon)$ including f-b as well as f-f from a specified $F_{1}(E)$ and evaluate the $F_{2}(E)$ which would be erroneously inferred by solving the inverse problem ignoring the presence of the f-b term, as is currently done in all HXR data analysis, whether by inversion or forward fitting.

By (4) and (11) the total $\mathrm{f}-\mathrm{f}+\mathrm{f}-\mathrm{b}$ emission spectrum $\mathrm{d} J / \mathrm{d} \epsilon$ from a homogeneous volume $V$ can be written

$H(\epsilon)=\int_{\epsilon}^{\infty} G(E) \mathrm{d} E+D \Sigma_{Z_{\mathrm{eff}} \leq \sqrt{\epsilon / \chi}} Z_{\mathrm{eff}}^{4} A_{Z_{\mathrm{eff}}} G\left(\epsilon-V_{Z_{\mathrm{eff}}}\right)$,

where

$H(\epsilon)=\frac{3}{8 \alpha r_{\mathrm{e}}^{2}} \frac{1}{\zeta_{\mathrm{B}} m_{\mathrm{e}} c^{2} n_{\mathrm{p}} V} \epsilon \frac{\mathrm{d} J}{\mathrm{~d} \epsilon} ; \quad G(E)=F(E) / E$

and $D$ is as given in Eq. (A.2). If we ignore the second (recombination) term in Eq. (20), as has always been done in the past, for the Kramers f-f term, the inverse is just (Brown \& Emslie 1988)

$G(\epsilon)=-H^{\prime}(E)$.

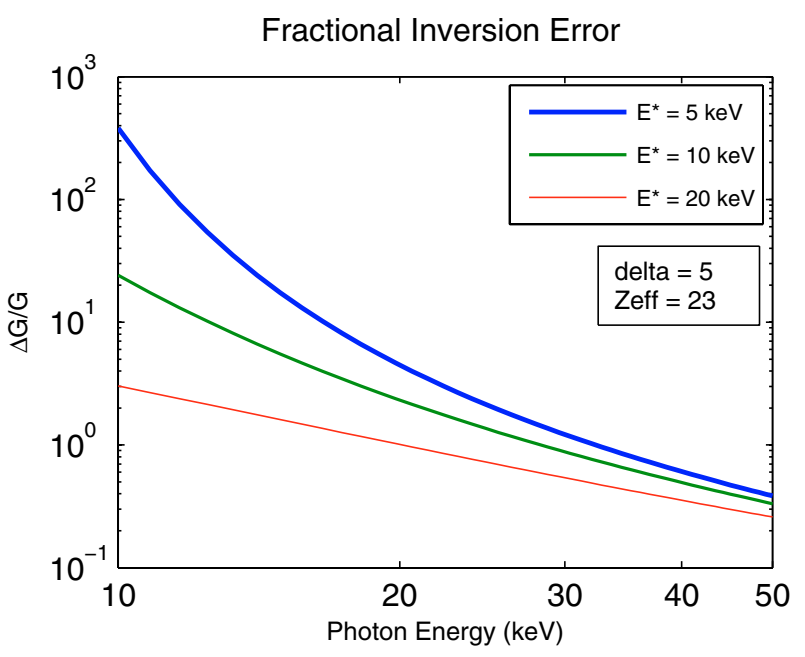

Fig. 6. Fractional error $(\Delta G / G)$ in $\mathrm{G}$ (Eq. (25)) as discussed in Sect. 5 for $E_{*}=5,10,20 \mathrm{keV}$ respectively for a shifted power-law due to inference of $\mathrm{G}$ from $\mathrm{H}$ ignoring the presence of recombination.

The neglect of the second term can be thought of as an "error" $\Delta H$ in our data and if we apply inversion formula (22) to this "data", ignoring the recombination "error" we get a resulting error $\Delta G$ in the inferred $G$ given by

$$
\begin{aligned}
\Delta G(E) & =\frac{F_{2}(E)-F_{1}(E)}{E} \\
& =-D \Sigma_{Z_{\mathrm{eff}} \leq \sqrt{\epsilon \chi \chi}} Z_{\mathrm{eff}}^{4} A_{Z_{\mathrm{eff}}} G^{\prime}\left(E-V_{Z_{\mathrm{eff}}}\right) .
\end{aligned}
$$

It is at once clear that any sharp change in $j(\epsilon)$ i.e. in $H(E)$, such as the presence of $\mathrm{f}-\mathrm{b}$ edges, however small, can have a very large effect on the inferred $F_{2}(E)$. (If the inverse problem is addressed for more realistic smoother forms of $\mathrm{f}-\mathrm{f}$ cross section than Kramers, the "error magnification" is in general even larger - Brown \& Emslie 1988; Piana et al. 2000.) For a power law $F$ with cut off around say $20 \mathrm{keV}$, analytically speaking this expression gives infinite negatives in $\Delta G(E)$ at the spectral edges around $30 \mathrm{keV}$ (for $\mathrm{Fe}$ ). However when smoothed over a few $\mathrm{keV}$ and added to the f-f term the result would be a "wiggle" in the $F(E)$ solution in the $30-40 \mathrm{keV}$ range. This is just where enigmatic features have been reported in some RHESSI spectra and variously attributed to the effects of photospheric albedo (Kontar et al. 2006), possibly pulse pile up (Piana et al. 2003), or a high value of $E_{\mathrm{c}}$ (Zhang \& Huang 2004).

Another case providing insight is that of a smooth shifted power-law $G(E)=A\left(E+E_{*}\right)^{-\delta-1}$, which has no edges though the corresponding $F(E)$ has a smooth peak at $E=E_{*} / \delta$. In this case the fractional error in $G$ due to applying (22) ignoring the recombination term can be expressed as

$\frac{\Delta G(E)}{G(E)}=(\delta+1) \frac{D}{E+E_{*}} \Sigma_{Z_{\mathrm{eff}}} Z_{\mathrm{eff}}^{4} A_{Z_{\mathrm{eff}}}\left[\frac{1}{1-V_{Z_{\mathrm{eff}}} /\left(E+E_{*}\right)}\right]^{\delta+2}$,

where each term in the $Z_{\text {eff }}$ sum is zero for $E<V_{Z_{\text {eff }}}=Z_{\text {eff }}^{2} \chi$.

In the case of recombination onto $\mathrm{FeXXV}$ alone (hot plasma), this gives for $\delta=5$,

$\frac{\Delta G}{G} \approx \frac{10 \mathrm{keV}}{E+E_{*}}\left[1-7 \mathrm{keV} /\left(E+E_{*}\right)\right]^{-7}$,

which is shown in Fig. 6 for $E_{*}=5,10,20 \mathrm{keV}$. Evidently errors due to neglect of recombination can be large at low $E$. The reason is that the $Z_{\text {eff }}$ recombination contribution to the 
bremsstrahlung solution for $G(E)$ at $E$ comes from the slope of $G$, and not just $G$ itself and at $E-V_{Z_{\text {eff }}}$ not at $E$. Figure 6 is similar to Fig. A.2 because $F_{2} / F_{1}=G_{2} / G_{1}=1+\Delta G / G_{1}$.

This error has very serious consequences for past analyses of HXR flare spectra, at least in cases where a significant hot dense coronal loop is involved. For example, the $\mathrm{f}-\mathrm{b}$ emission spectrum is most important at lower energies (5-30 keV or so), depending on the plasma temperature $T$ and low energy electron cut-off or roll-over $E_{\mathrm{c}}, E_{*}$ and is steeper than the free-free. This will offset some of the spectral flattening caused around such energies by photospheric albedo (Alexander \& Brown 2003; Kontar et al. 2005) resulting in underestimation of the albedo contribution and hence of the downward beaming of the fast electrons. This fact would weaken the finding of Kontar \& Brown (2006) that the electrons are near isotropic, in contradiction of the usual thick target description, but for the fact that the flares they used had rather hard spectra and substantial footpoint emission - conditions where the f-b correction should be rather small. Nevertheless it illustrates that care is needed to ensure f-b emission is properly considered.

Finally, recognising the presence of the f-b contribution, one can in fact convert integral Eq. (20) into a differential/functional equation for $F(E)$ by differentiation, namely

$G(E)-D \Sigma_{Z_{\mathrm{eff}} \geq E / \chi^{1 / 2}} A_{Z_{\mathrm{eff}}} Z_{\mathrm{eff}}^{4} G^{\prime}\left(E-Z_{\mathrm{eff}}^{2} \chi\right)=-H^{\prime}(E)$,

which is a wholly new class of functional equation in need of exploration.

\section{Discussion and conclusions}

It is clear from our findings that ignoring non-thermal f-b contribution as negligible, as has been done in the past, is erroneous. Even if we ignore coronal enhancement of element abundances, and use photospheric abundances, f-b contribution can be very significant. In certain flaring regions, especially in densehot coronal sources or occulted loop-top events, fast electron recombination can be of vital importance in analysing data properly and in inferring electron spectra and energy budgets. It can have a major influence on inferred electron spectra both as an inverse problem and also in forward fitting parameters, including the important potential to find and evaluate low-energy electron cut-offs, which are vital to flare energy budgets. While incorporating $\mathrm{f}-\mathrm{b}$ into spectral fitting procedures will make it considerably more complicated, an advantage is that the f-b, unlike the $\mathrm{f}-\mathrm{f}$, contribution retains its $J(\epsilon)$ signatures of any sharp features in $F(E)$.

A major consequence of the low energy f-b contribution is that, to fit an actual photon spectrum, less electrons are needed, than in f-f only modelling, at the low $E$ end, which is where most of the power in $F(E)$ lies. For example, if we consider the case $\delta=5, E_{\mathrm{c}}=10 \mathrm{keV}$ and ionisation up to Fe XXV, then we see from Figs. 1 and 2 that inclusion of $\mathrm{f}-\mathrm{b}$ increases $j$ by a factor of 2-10 in the $15-20 \mathrm{keV}$ range for $\delta=3-5$. Thus, to get a prescribed $j$ in that range we need only $10-50 \%$ as many electrons as inferred from f-f emission only.

We also note that the importance of non-thermal $f-b$ emission is greatest when non-thermal electrons are present at low $E$ and with large $\delta$ such as in microflares with "hard" XRs in the few to ten KeV range (Krucker et al. 2002). Such low energy electrons have short collisional mfps and so are more likely to emit mainly in hot coronal regions, if accelerated there. Microflares are therefore important cases for inclusion of $\mathrm{f}-\mathrm{b}$.

Before we conduct any precise fitting of $F(E)$, involving the $\mathrm{f}-\mathrm{b}$ contribution, to real data (e.g. from RHESSI) and include it in software packages it will be important to include, for both f-b and f-f, more accurate cross-sections with Gaunt factors etc. and ionisation fractions as functions of plasma temperature. By doing this, it will be possible to show, for certain events, how vital recombination is and to improve our understanding of electron spectra and their roles in flares. However, our Kramers results already bring out the fact that recombination should not be ignored in the future, and that it may be invaluable in some cases as a diagnostic of the presence or otherwise of electron spectral features.

Acknowledgements. This work was supported by a PPARC Rolling Grant and UC Berkeley Visitor funds (J.C.B.) and by a Dorothy Hodgkin's Scholarship (P.C.V.M.). Helpful discussions with A. Caspi, H. S. Hudson, A. G. Emslie and J. Kasparova are much appreciated, as are the helpful suggestions of the referee (S. Krucker).

\section{Appendix A: Efficiency and smooth $F(E)$}

\section{A.1. Comparison of the efficiency of $f-b$ versus $f-f H X R$ yield}

In Sects. 3.1 and 3.2, we predicted the $j_{\mathrm{B}}, j_{\mathrm{R}}$ from a power-law $F(E)$ and found that the $j_{\mathrm{R}}$ contribution could sometimes be more important than $j_{\mathrm{B}}$. It is of interest therefore, to consider the following question. If one observes a power-law $j(\epsilon) \propto \epsilon^{-\gamma}$ above some $\epsilon \geq V_{Z}$, what electron flux $F_{\mathrm{R}}(E)$ would be needed to generate it in a plasma of solar abundances purely by nonthermal electron recombination on ion $Z_{\mathrm{eff}}$ as compared with the $F_{\mathrm{B}}(E)$ required to do so purely by $\mathrm{f}-\mathrm{f}$ bremsstrahlung? If we write, from Eq. (15), the latter as $F_{\mathrm{B}}(E)=C E^{-\gamma+1}$ then the former has to be, by Eq. (16),

$F_{\mathrm{R}}(E)=C\left(E+V_{Z_{\mathrm{eff}}}\right)^{-\gamma} / D Z_{\mathrm{eff}}^{4} A_{Z_{\mathrm{eff}}}$,

where

$D=\frac{2 \pi \chi}{\sqrt{3} \zeta_{\mathrm{B}}} \approx 0.04 \mathrm{keV}$

and the ratio measuring recombination efficiency relative to bremsstrahlung is

$\frac{F_{\mathrm{B}}(E)}{F_{\mathrm{R}}(E)}=\gamma Z_{\mathrm{eff}}^{4} A_{Z_{\mathrm{eff}}} \frac{D}{E}\left[1+V_{Z_{\mathrm{eff}}} / E\right]^{\gamma}$

which we show in Fig. A.1 for $\gamma=5$ in terms of each of the dominant $\mathrm{f}-\mathrm{b}$ contributions from fully ionised $\mathrm{O}, \mathrm{Mg}, \mathrm{Si}$ and Fe respectively while the $f-f$ is for all elements. Evidently nonthermal recombination could be dominant over bremsstrahlung up to many $10 \mathrm{~s}$ of $\mathrm{keV}$ as the most efficient HXR source if the electrons are emitted entirely in a plasma hot enough ( $T \approx 20 \mathrm{MK}$ ) for elements up to Fe $24+$ to be ionised and is significant even at lower temperatures.

In terms of the total required electron fluxes $F_{\mathrm{R} 1}, F_{\mathrm{B} 1}$ above energy $E_{1}$, the ratio is

$$
\begin{aligned}
\frac{F_{\mathrm{B} 1}}{F_{\mathrm{R} 1}} & =\frac{\gamma-1}{\gamma-2} Z_{\mathrm{eff}}^{4} A_{Z_{\mathrm{eff}}} \frac{D}{E_{1}}\left[1+V_{Z_{\mathrm{eff}}} / E_{1}\right]^{\gamma-1} \\
& \approx 0.02 Z_{\mathrm{eff}}^{4} A_{Z_{\mathrm{eff}}} \frac{10 \mathrm{keV}}{E_{1}}\left[1+V_{Z_{\mathrm{eff}}} / E_{1}\right]^{\gamma-1}
\end{aligned}
$$

which is about 10 for $\mathrm{Fe}, 0.25$ for $\mathrm{Si}$ and 0.1 for $\mathrm{Mg}$ and $\mathrm{O}$ at $E_{1}=10 \mathrm{keV}$.

At higher electron energies ( $E \geq \approx 17 \mathrm{keV}$ ), O becomes more efficient than $\mathrm{Mg}$, as can be seen in Fig. A.1, because of the combined effects of the $A_{Z} Z^{4}$ factor and the term containing $V_{Z}$. 


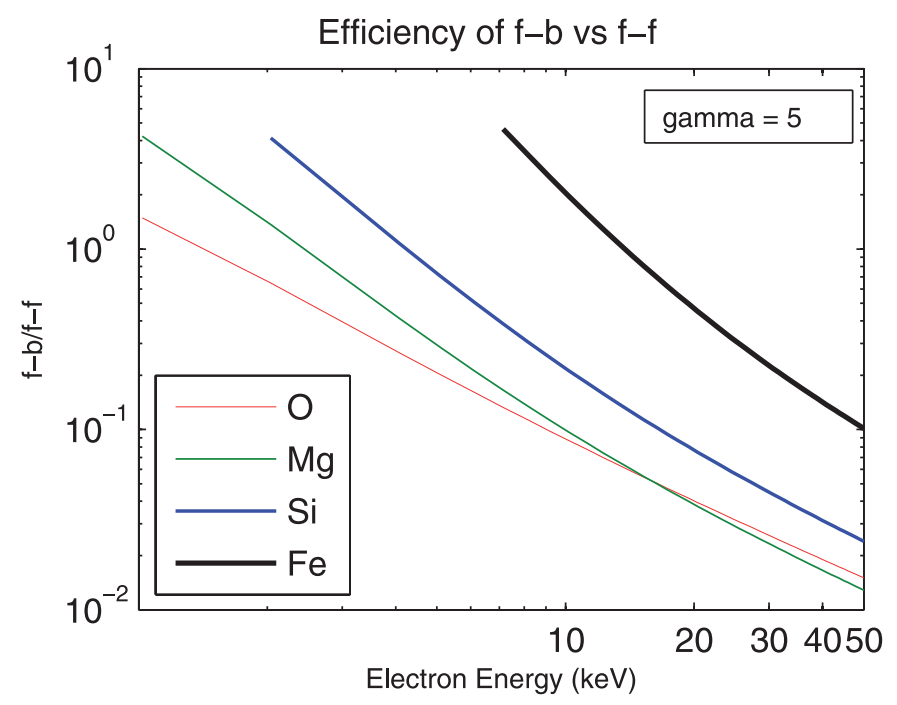

Fig. A.1. The f-b electron efficiency compared to f-f for the 4 elements discussed in Appendix A. It is evident from the graph that, if present, highly ionised $\mathrm{Fe}$ is the most efficient source of $\mathrm{f}-\mathrm{b}$ HXRs in terms of the $F(E)$ needed followed by $\mathrm{Si}, \mathrm{O}$ and $\mathrm{Mg}$.

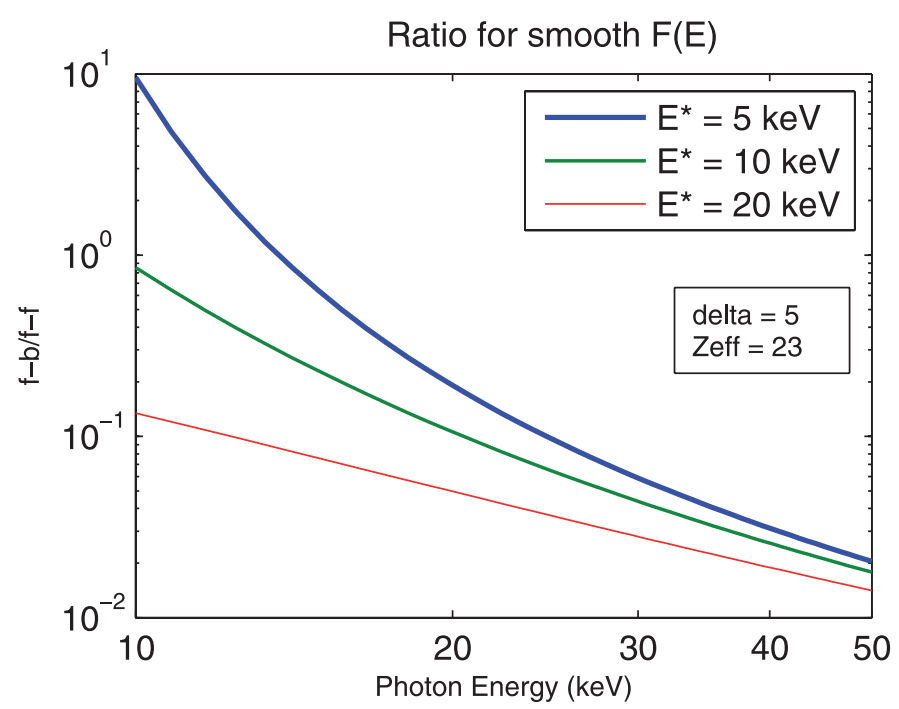

Fig. A.2. The $\Psi_{\text {smooth }}$ as discussed in Appendix A and Eq. (A.5). It is the ratio of $j_{\mathrm{R}}$ to $j_{\mathrm{B}}$ for the smooth $F(E) \propto E\left(E+E_{*}\right)^{-\delta-1}$ for $E_{*}=$ $5,10,20 \mathrm{keV}$ respectively.

\section{A.2. Ratio of $j_{R}$ to $j_{B}$ for an example of a smooth $F(E)$ with no cut-off}

All of the above results are for $F(E)$ with a sharp cut off $E_{\mathrm{c}}$. To illustrate how the appearance of $j(\epsilon)$ is modified by inclusion of $\mathrm{f}-\mathrm{b}$ as well as f-f for a smooth $F(E)$, a simple case to evaluate is $F(E) \propto E\left(E+E_{*}\right)^{-\delta-1}$, which behaves as $E^{-\delta}$ at $E \gg E_{*}$ but has a smooth roll-over at $E_{*} / \delta$. It is simple to show that the resulting $j_{\mathrm{B}}(\epsilon) \propto\left(E+E_{*}\right)^{-\delta} / \delta$ for $\mathrm{f}-\mathrm{f}$ alone and that the ratio of $\mathrm{f}-\mathrm{b}$ to $\mathrm{f}-\mathrm{f}$ in this case is, for ion $Z_{\mathrm{eff}}$ alone,

$\Psi_{\text {smooth }}=\frac{D \zeta_{Z_{\text {eff }}}}{\epsilon+E_{*}}\left[1-\frac{Z_{\mathrm{eff}}^{2}}{\epsilon+E_{*}}\right]^{-\delta-1}$,

which is shown in Fig. A.2 for $\delta=5, Z_{\text {eff }}=23.77$ and $E_{*}=$ $5,10,20 \mathrm{keV}$. We see again that $\Psi_{\text {smooth }}$ is largest for large $\delta$ and for small $E_{*}$.

\section{Appendix B: Whole flare thin target, thermal, and thick target expressions for f-f and f-b HXR emission spectra}

Here we extend the above results on local emissivities $j(\epsilon)$ to estimate total spectral emission rate $J(\epsilon)$ (photons s ${ }^{-1}$ per unit $\epsilon$ ) from extended flare volumes as required for real flare data.

\section{B.1. Thin target coronal loop}

A thin target is one in which $F(E)$ is not significantly modified by energy losses or gains over the volume. For a loop of half length $L$, transverse area $A$, volume $2 A L$ and density $n_{\mathrm{p}}$, the total emission rate spectra contributions $J_{\text {thin }}(\epsilon)$ are for a power law $F(E)$ with a low energy cut-off, by Eq. (15),

$$
\begin{aligned}
J_{B_{\text {thin }}}(\epsilon)= & \frac{\delta-1}{\delta} \frac{8 \alpha \zeta_{\mathrm{B}}}{3} \frac{m_{\mathrm{e}} c^{2} r_{\mathrm{e}}^{2}}{\epsilon} \frac{2 n_{\mathrm{p}} A L F_{\mathrm{c}}}{E_{\mathrm{c}}} \\
& \times\left[\frac{\epsilon}{E_{\mathrm{c}}}\right]^{-\delta} ; \epsilon \geq E_{\mathrm{c}} \\
& 1 ; \epsilon<E_{\mathrm{c}}
\end{aligned}
$$

and by Eq. (16),

$$
\begin{aligned}
J_{\mathrm{R}_{\mathrm{thin}}}(\epsilon)= & (\delta-1) \frac{32 \pi \zeta_{\mathrm{R} Z_{\mathrm{eff}}}}{3^{1 / 2} \alpha} \frac{r_{\mathrm{e}}^{2} \chi}{\epsilon} \frac{2 n_{\mathrm{p}} A L F_{\mathrm{c}}}{E_{\mathrm{c}}^{2}} \times \Sigma_{Z_{\mathrm{eff}}} \\
& \times\left[\frac{\epsilon-Z_{\mathrm{eff}}^{2} \chi}{E_{\mathrm{c}}}\right]^{-\delta-1} ; \epsilon \geq E_{\mathrm{c}}+Z_{\mathrm{eff}}^{2} \chi \\
& \times 0 ; \epsilon<E_{\mathrm{c}}+Z_{\mathrm{eff}}^{2} \chi,
\end{aligned}
$$

where the summation is over all $Z_{\mathrm{eff}} \leq\left(\epsilon-E_{\mathrm{c}}\right]^{1 / 2}$. These spectral shapes $J(\epsilon)$ are of course just the same as the thin target $j$ forms, scaled by the plasma volume.

\section{B.2. Hot coronal loop thermal emission (in the Kramers approximation)}

Both f-f and f-b emissions are included in the standard analyses (e.g. Mewe et al. 1987; Dere et al. 1996) of isothermal hot plasma contributions to flare spectra, using full cross sections and ionisation balance expressions. It is therefore surprising that $\mathrm{f}-\mathrm{b}$ is omitted from calculations of non-thermal emission, especially at low $\epsilon$, where electrons of comparable energy are present in both thermal and non-thermal populations. In applying our study of the non-thermal f-b to real data we wish to include thermal emission as it is important at energies under about $20 \mathrm{keV}$ and so dilutes the visibility of non-thermal contributions. In order to treat the thermal and non-thermal $j$ consistently and allow meaningful comparisons we use the expressions for the thermal $j$ relevant to the Kramers cross sections just as in the non-thermal case - but see remarks previously and below concerning Gaunt factors and absolute accuracy of our results.

For an isothermal plasma the local Maxwellian electron flux spectrum is

$F_{\text {therm }}(E)=\left[\frac{8}{\pi m_{\mathrm{e}}}\right]^{1 / 2} \frac{E}{(k T)^{3 / 2}} n_{\mathrm{p}} \exp (-E / k T)$,

which, by Eq. (4), gives for the thermal bremsstrahlung emission from a uniform loop

$J_{\mathrm{Btherm}}(\epsilon)=\frac{16 \alpha r_{\mathrm{e}}^{2}}{3} \zeta_{\mathrm{B}} m_{\mathrm{e}} c^{2} \times\left[\frac{8}{\pi m_{\mathrm{e}}}\right]^{1 / 2} \frac{2 n_{\mathrm{p}}^{2} A L \mathrm{e}^{-\epsilon / k T}}{\epsilon(k T)^{1 / 2}}$ 
Non-thermal $f-b$ and $f-f$
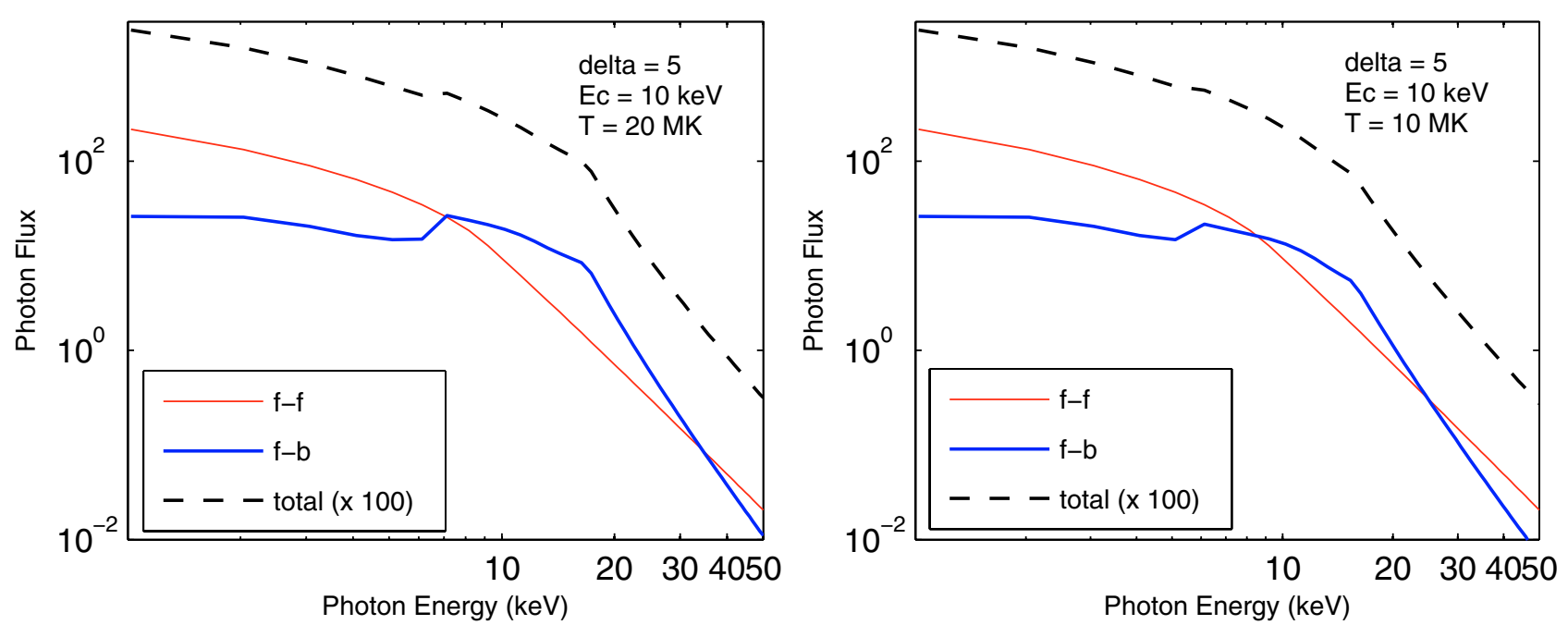

Fig. B.1. Non-thermal f-f and f-b spectra for the thick target case (Eqs. (B.11) and (B.12)) shown for 2 different temperatures: 20 MK that is pertinent to events such as the 2002 April 14 event and $10 \mathrm{MK}$, which is more in the range of "microflare" temperatures. It is interesting to note the three distinct energy regimes for the f-b spectrum, namely: $\epsilon<V_{\mathrm{Fe}} ; V_{\mathrm{Fe}} \leq \epsilon \leq V_{\mathrm{Fe}}+E_{\mathrm{c}} ; \epsilon>V_{\mathrm{Fe}}+E_{\mathrm{c}}$. Clearly f-b is very important in the $10-50 \mathrm{keV}$ range, precisely where albedo issues are also important.

and for the recombination

$J_{\text {Rtherm }}(\epsilon)=$

$$
\sqrt{\frac{2 \pi}{27 m_{\mathrm{e}}}} \frac{64 r_{\mathrm{e}}^{2} \chi^{2}}{\alpha} \frac{2 n_{\mathrm{p}}^{2} A L}{\epsilon(k T)^{3 / 2}} \Sigma_{Z_{\mathrm{eff}}} \zeta_{\mathrm{R} Z_{\mathrm{eff}}} \exp \left(\frac{Z_{\mathrm{eff}}^{2} \chi-\epsilon}{k T}\right) .
$$

These results can be compared with those of Culhane (1969); Culhane \& Acton (1970) who were among the first to explicitly address the X-ray spectrum from hot coronal plasmas. Using the Kramers cross sections is essentially equivalent to setting to unity all Gaunt factors in their expressions. When we do so, the $\epsilon, T$ dependences of our $J_{\text {Rtherm }}, J_{\text {Btherm }}$ are identical to theirs e.g. $J_{\text {Rtherm }} / J_{\text {Btherm }}$ is independent of $\epsilon$, the only difference being that our $J_{\text {Rtherm }}$ is much larger (in absolute value) than theirs, mainly because they used the very much lower value of $A_{Z}$ for Fe believed at that time. Examination of the $\epsilon, T$ dependences of Culhane's Gaunt factors shows that they affect quite significantly both the f-f and the f-b spectra from a Maxwellian $F(E)$ and we should expect the same to be true for non-thermal $F(E)$ like power-laws. Thus, any accurate absolute comparison of predictions with data will require incorporation of appropriate $g, G$. However, these do not affect the absolute orders of magnitude of $J_{\text {Rtherm }}, J_{\text {Btherm }}$ nor the dependencies on $n_{\mathrm{p}}, V, F_{\mathrm{c}}$ etc., nor the locations of edges. So, for the present purpose of demonstrating the importance of $f-b$, the Kramers expressions will suffice.

\section{B.3. Thick target (dense loop or footpoint) $f-f$ and $f-b$ emission spectra}

In the thick target case, $j$ evolves in space along with the energy losses of the electrons. To find $j$ locally one uses the continuity equation (Brown 1972) and then integrates over volume to get $J$. However, to get the whole volume $J$, it is actually simpler (Brown 1971) to start with the electron injection rate spectrum $\mathcal{F}_{\mathrm{o}}\left(E_{\mathrm{o}}\right)$ electrons/s per unit injection energy $E_{\mathrm{o}}$ and use the expression

$J_{\text {thick }}(\epsilon)=\int_{E_{\mathrm{o}}} \mathcal{F}_{\mathrm{o}}\left(E_{\mathrm{o}}\right) \eta\left(\epsilon, E_{\mathrm{o}}\right) \mathrm{d} E_{\mathrm{o}}$, where $\eta\left(\epsilon, E_{\mathrm{o}}\right)$ is the total number of photons per unit $\epsilon$ emitted by an electron of energy $E_{\mathrm{o}}$ as it decays in energy. For purely collisional losses $\mathrm{d} E / \mathrm{d} N=-K / E$ with $K=2 \pi e^{4} \Lambda, e$ being the electronic charge and $\Lambda$ the Coulomb Logarithm. Then

$\eta\left(\epsilon, E_{\mathrm{o}}\right)=\frac{1}{K} \int_{E} E \frac{\mathrm{d} Q}{\mathrm{~d} \epsilon} \mathrm{d} E$

for the relevant radiation cross section $\mathrm{d} Q / \mathrm{d} \epsilon$. Note that this assumes $\mathrm{H}$ to be uniformly and fully ionised along the electron path. For partially ionised $\mathrm{H}$ the energy loss constant $K$ is reduced but this situation is not relevant to our hot source situations.

For our Kramers $\mathrm{d} Q / \mathrm{d} \epsilon$ f-f and f-b expressions (3), (7) and (9), the resulting expressions, in the case where $A_{Z_{\mathrm{eff}}}$ are uniform along the path, Eq. (B.7) gives

$$
\begin{aligned}
\eta_{\mathrm{B}}\left(\epsilon, E_{\mathrm{o}}\right)= & \frac{8 \alpha \zeta_{\mathrm{B}}}{3} \frac{r_{\mathrm{e}}^{2} m_{\mathrm{e}} c^{2}}{K} \\
& \times\left[\frac{E_{\mathrm{o}}}{\epsilon}-1\right] ; \epsilon \leq E_{\mathrm{o}} \\
& 0 ; \epsilon>E_{\mathrm{o}}
\end{aligned}
$$

and

$$
\begin{aligned}
\eta_{\mathrm{RZ}}\left(\epsilon, E_{\mathrm{o}}\right)= & \frac{32 \pi A_{Z_{\mathrm{eff}}} Z_{\mathrm{eff}}^{4}}{3^{3 / 2} \alpha} \frac{r_{\mathrm{e}}^{2} \chi^{2}}{K \epsilon} \\
& \times 1 ; E_{\mathrm{o}} \geq \epsilon+Z_{\mathrm{eff}}^{2} \chi \\
& 0 ; E_{\mathrm{o}}<\epsilon+Z_{\mathrm{eff}}^{2} \cdot
\end{aligned}
$$

For a power-law injection rate spectrum of spectral index $\delta_{\mathrm{o}}$, viz

$\mathcal{F}_{\mathrm{o}}\left(E_{\mathrm{o}}\right)=\left(\delta_{\mathrm{o}}-1\right) \frac{\mathcal{F}_{\mathrm{oc}}}{E_{\mathrm{oc}}}\left[\frac{E_{\mathrm{o}}}{E_{\mathrm{oc}}}\right]^{-\delta_{\mathrm{o}}} ; E_{\mathrm{o}} \geq E_{\mathrm{oc}}$, 
where $\mathcal{F}_{\mathrm{oc}}$ is the total rate above low energy cut-off $E_{\mathrm{oc}}$, the expressions for the non-thermal emission spectra are then by Eq. (B.6)

$$
\begin{aligned}
J_{\text {Bthick }}(\epsilon)= & \frac{8 \alpha r_{\mathrm{e}}^{2}}{3} \frac{\zeta_{\mathrm{B}} m_{\mathrm{e}} c^{2} \mathcal{F}_{\mathrm{oc}}}{\left(\delta_{\mathrm{o}}-1\right)\left(\delta_{\mathrm{o}}-2\right) K} \\
& \times\left(\frac{\epsilon}{E_{\mathrm{c}}}\right)^{-\delta_{\mathrm{o}}+1} ; \epsilon \geq E_{\mathrm{c}} \\
& \times\left[\left(\delta_{\mathrm{o}}-1\right) \frac{E_{\mathrm{c}}}{\epsilon}-\left(\delta_{\mathrm{o}}-2\right)\right] ; \epsilon<E_{\mathrm{c}}
\end{aligned}
$$

and, for ion $Z_{\mathrm{eff}}$,

$$
\begin{aligned}
J_{\mathrm{R} Z_{\mathrm{eff}} \text { thick }}(\epsilon)=\frac{32 \pi r_{\mathrm{e}}^{2} m_{\mathrm{e}} c^{2}}{3^{3 / 2} \alpha} \zeta_{\mathrm{R} Z_{\mathrm{eff}}} \frac{\chi^{2}}{K \epsilon} \frac{\mathcal{F}_{\mathrm{oc}}}{E_{\mathrm{oc}}} & \\
& \times\left[\frac{\epsilon-Z_{\mathrm{eff}}^{2} \chi}{E_{\mathrm{oc}}}\right]^{-\delta_{\mathrm{o}}+1} ; \quad \epsilon \geq E_{\mathrm{oc}}+Z_{\mathrm{eff}}^{2} \chi \\
& \times\left[\frac{E_{\mathrm{oc}}-Z_{\mathrm{eff}}^{2} \chi}{E_{\mathrm{oc}}}\right]^{-\delta_{\mathrm{o}}+1} ; \\
0 ; & Z_{\mathrm{eff}}^{2} \chi<\epsilon<E_{\mathrm{oc}}+Z_{\mathrm{eff} \chi}^{2} \chi \\
& \epsilon<Z_{\mathrm{eff}}^{2} \chi .
\end{aligned}
$$

For the case of a cold thick target footpoint the total $\zeta_{\mathrm{R}}$ can be almost as small as 1 if only hydrogen and some low $\zeta_{\mathrm{R}}$ elements are ionised and even zero if $T<8000 \mathrm{~K}$ or so (there being almost no charged ions present). In these sources the f-b contribution is negligible or at most a very small correction. For a collisonally thick hot loop $\zeta_{\mathrm{R}}$ is, however, very much higher.

The main distinction of these hot thick target spectra compared to hot thin targets is that the decay of all electrons to zero energy means that the signature of the cut off $E_{\mathrm{oc}}$ in the injection spectrum appears not as a discontinuity in $J(\epsilon)$ but only in its gradient $J^{\prime}(\epsilon)$. This gradient break is very noticeable in Fig. B. 1 at energy $\epsilon=E_{\mathrm{c}}+V_{\mathrm{Fe}}$. So, even in the thick target case, spectral diagnosis of any $E_{\mathrm{oc}}$ present is possible. The recombination edges themselves appear at the relevant ionisation energies $\epsilon=V_{\text {eff }}$, these being from thick target electrons decelerated to zero $E$. These non-thermal recombination spectral edges are then down in the energy regime below $10 \mathrm{keV}$ which is complicated by Fe lines etc., making the interpretation of $\mathcal{F}_{\mathrm{o}}$ there, and of the lines, more difficult.

\section{References}

Andersen, L. H., Pan, G-Y., \& Schmidt, H. T. 1992, J. Phys., B, 25, 277 Arnaud, M., \& Raymond, J. 1992, ApJ, 398, 394

Arnoldy, R. L., Kane, S. R., \& Winckler, J. R. 1968, ApJ, 151, 711

Brown, J. C. 1971, Sol. Phys., 18,489

Brown, J. C. 1972, Sol. Phys., 26, 441

Brown, J. C. 2005, Solar Magnetic Phenomena, Proceedings of the 3rd Summerschool and Workshop held at the Solar Observatory Kanzelh ohe, K arnten, Austria, August 25-September 5, 2003, ed. A. Hanslmeier, A. Veronig, \& M. Messerotti, Astronomy and Astrophysics Space Science Library, 320 (Dordrecht, The Netherlands: Springer), 87, 87

Brown, J. C., \& Emslie, A. G. 1988, ApJ, 331, 164

Brown, J. C., Emslie, A. G., Holman, G. E., et al. 2006, ApJ, 643, 523

Conway, A. J., Brown, J. C., Eves, B. A. C., \& Kontar, E. P. 2003, A\&A, 407, 725

Craig, I. J. D., \& Brown, J. C. 1986, Inverse Problems in Astronomy (New York and London: IoPP Adam Hilger)

Culhane, J. L. 1969, MNRAS, 144, 375

Culhane, J. L., \& Acton, L. W. 1970, MNRAS, 151, 141

Dere, K. P., Landi, E., Mason, H. E., Monsignori Fossi, B. C., \& Young, P. R. 1997, A\&AS, 125, 149

Erdos, A., Mezzorani, G., \& Quarati, P. 1993, Phys. Rev. A, 48,452

Feldman, U., Mandelbaum, P., Seely, J. L., Doschik, G. A., \& Garsky, H. 1992, ApJ, 81, 387

Hahn, Y. 1997, Rep. Prog. Phys., 60, 691

Hahn, Y., \& Krstic, P. 1994, J. Phys., B 27, L509

Johns-Krull, C., \& Lin, R. P. 1992, Sol. Phys., 137, 121

Kasparová, J., Kontar, E. P., \& Brown, J. C. 2007, A\&A, 466, 705

Koch, H. W., \& Motz, J. W. 1959, Rev. Mod. Phys., 31, 4, 920

Kontar, E. P., Emslie, A. G., Piana, M., Massone, A. M., \& Brown, J. C. 2005, Sol. Phys., 226, 137

Kontar, E. P., MacKinnon, A. L., Schwartz, R. A., \& Brown, J. C. 2006, A\&A, 446,1157

Korchak, A. A. 1971, Sol. Phys., 18, 284

Kramers, H. A. 1923, Phil. Mag., 46, 836

Krucker, S., Christe, S., Lin, R. P., Hurford, G. J., \& Schwartz, R. A. 2002, Sol. Phys., 210, 445

Landini, M., Monsignori, F. B. C., \& Pallavicini, R. 1973, Sol. Phys., 29, 93

Lin, R. P., \& Schwartz, R. A. 1987, ApJ, 312, 462

Lin, R. P., Dennis, B. R., Hurford, G. J., et al. 2002, Sol. Phys., 210, 3

MacKinnon, A. L. 2006, in Solar Eruptions and Energetic Particles, ed. N. Gopalswamy, R. Mewaldt, \& J. Torsti, AGU Geophysical Monograph, 165, 157

Mallik, P. C. V., \& Brown, J. C. 2007, RAS NAM (Preston), Abstract P20.6

Massone, A. M., Emslie, A. G., Kontar, E. P., et al. 2004, ApJ, 613, 1233

Mewe, R., Lemen, J. R., \& van den Oord, G. H. J. 1986, A\&AS, 65, 511

Piana, M., Massone, A. M., Kontar, E. P., et al. 2003, ApJ, 595 , L127

Piana, M., Barrett, R. K., Brown, J. C., \& McIntosh, S. W. 2000, Inverse Problems, 15, 1469

Veronig, A., \& Brown, J. C. 2004, ApJ, 603, L117

Vilmer, N., MacKinnon, A. L., Trottet, G., \& Barat, C. 2003, A\&A, 412, 865

Zhang, J., \& Huang, G. L. 2004, Sol. Phys., 219, 135 